\title{
Testing the doubly charged charm-strange tetraquarks
}

\author{
S. S. Agaev ${ }^{1,2}$, K. Azizi ${ }^{3,4, a}$, H. Sundu ${ }^{1}$ \\ ${ }^{1}$ Department of Physics, Kocaeli University, 41380 Izmit, Turkey \\ ${ }^{2}$ Institute for Physical Problems, Baku State University, 1148 Baku, Azerbaijan \\ ${ }^{3}$ Department of Physics, Doğuş University, Acibadem-Kadiköy, 34722 Istanbul, Turkey \\ ${ }^{4}$ School of Physics, Institute for Research in Fundamental Sciences (IPM), P. O. Box 19395-5531, Tehran, Iran
}

Received: 5 December 2017 / Accepted: 10 February 2018 / Published online: 19 February 2018

(C) The Author(s) 2018. This article is an open access publication

\begin{abstract}
The spectroscopic parameters and decay channels of the doubly charged scalar, pseudoscalar and axialvector charm-strange tetraquarks $Z_{\bar{c} s}=[s d][\overline{u c}]$ are explored within framework of the QCD sum rule method. The masses and current couplings of these diquark-antidiquark states are calculated by means of two-point correlation functions and taking into account the vacuum condensates up to eight dimensions. To compute the strong couplings of $Z_{\bar{c} s}$ states with $D, D_{s}, D^{*}, D_{s}^{*}, D_{s 1}(2460), D_{s 0}^{*}(2317), \pi$ and $K$ mesons we use QCD light-cone sum rules and evaluate width of their $S$ - and $P$-wave decays to a pair of negatively charged conventional mesons: For the scalar state $Z_{\bar{c} s} \rightarrow D_{s} \pi, D K, D_{s 1}(2460) \pi$, for the pseudoscalar state $Z_{\bar{c} s} \rightarrow D_{s}^{*} \pi, D^{*} K, D_{s 0}^{*}(2317) \pi$, and for the axial-vector state $Z_{\bar{c} s} \rightarrow D_{s}^{*} \pi, D^{*} K, D_{s 1}(2460) \pi$ decays are investigated. Obtained predictions for the spectroscopic parameters and decay widths of the $Z_{\bar{c} s}$ tetraquarks may be useful for experimental investigations of the doubly charged exotic hadrons.
\end{abstract}

\section{Introduction}

During last decade tetraquarks, i.e. bound states of four quarks are in the center of intensive experimental and theoretical investigations. Starting from discovery of the famous resonance $X(3872)$ in $B$ meson decay $B \rightarrow K X \rightarrow$ $K J / \psi \rho \rightarrow K J / \psi \pi^{+} \pi^{-}$by Belle [1], and after observation of the same state by other groups [2-4] experimental collaborations collected valuable information on the spectroscopic parameters and decay channels of the exotic states. They were discovered in various inclusive and exclusive hadronic processes. In this connection it is worth to note $B$ meson decays, $e^{+} e^{-}$and $\bar{p} p$ annihilations and $p p$ collisions. Theoretical studies of exotic hadrons, apart from tetraquark states,

\footnotetext{
a e-mail: azizi.hep.ph@gmail.com
}

include pentaquarks and hybrid mesons and encompass variety of models and calculational methods claiming to explain the internal structure of these states and calculate their experimentally measured parameters. Comprehensive information on collected experimental data and detailed analysis of theoretical achievements and existing problems can be found in latest review works Refs. [5-9].

The great success in physics of the exotic hadrons is connected with discovery of charged multiquark resonances. The first charged tetraquarks, namely $Z^{ \pm}(4430)$ states were observed by the Belle Collaboration in $B$ meson decays $B \rightarrow$ $K \psi^{\prime} \pi^{ \pm}$as resonances in the $\psi^{\prime} \pi^{ \pm}$invariant mass distributions [10]. The resonances $Z^{+}(4430)$ and $Z^{-}(4430)$ were detected and studied by Belle in the processes $B \rightarrow K \psi^{\prime} \pi^{+}$ [11] and $B^{0} \rightarrow K^{+} \psi^{\prime} \pi^{-}$[12], as well. These states constitute an important subclass of multiquark systems, because charged resonances can not be explained as excited charmonium or bottomonium states, and therefore, are real candidates to genuine tetraquarks.

Hadrons built of four quarks of different flavors form another intriguing class in the tetraquark family. Depending on a quark content these states may be neutral or charged particles. Among the observed tetraquarks the $X(5568)$ resonance remains a unique candidate to a hadron composed of four different quarks. At the same time it is a particle containing $b$-quark, i.e. is an open bottom tetraquark. The evidence for $X(5568)$ was first reported by the D0 Collaboration in Ref. [13]. Later it was observed again by D0 in the $B_{s}^{0}$ meson's semileptonic decays [14] . But other experimental groups, namely the $\mathrm{LHCb}$ and CMS collaborations could not find this resonance from analysis of their experimental data $[15,16]$, which make the experimental situation around $X(5568)$ unclear and controversial. Numerous theoretical works devoted to investigation of $X(5568)$ resonance's structure and calculation of its parameters led also to contradictory conclusions. The results of these studies are in a reasonable agreement with measurements carried out 
by the D0 Collaboration, while in other works an existence of the $X(5568)$ state is an object of discussions [17-33] . The detailed analysis of problems related to the status of the $X(5568)$ resonance can be found in original papers (see for instance, Ref. [6] and references therein).

The tetraquarks which might carry double electric charge constitute another interesting class of exotic hadrons [34]. These hypothetical particles if observed can be interpreted as diquark-antidiquark states: Formation of molecular states from two mesons of same charge is almost impossible due to repulsive forces between them. The doubly charged particles may exist, for example, as double charmed tetraquarks $[c c][\bar{d} \bar{s}]$ or $[c c][\bar{s} \bar{s}]$. In other words, they may contain two or three quark flavors. The phenomenology of these states, their decay modes and production mechanisms were investigated in Ref. [34]. In the context of the lattice QCD the mass spectra of these particles were evaluated in the paper [35]. As it was revealing recently the tetraquarks containing quarks of four different flavors may also carry double electric charge [36]. In fact, it is not difficult to see that tetraquarks $Z_{\bar{c} s}=[s d][\overline{u c}]$ and $Z_{c \bar{s}}=[u c][\bar{s} \bar{d}]$ belong to this category of particles, and at the same time, are open charm states. Authors of Ref. [36] wrote down also possible $S$ - and $P$-wave decay channels of these states. Strictly speaking, the open charm tetraquarks were previously investigated in the literature (see, for example Refs. [37-39]). The spectroscopic parameters and decay widths of the open charm tetraquark containing three different light quarks were calculated in Ref. [38]. In this study the open charm tetraquark was considered as a partner of the $X(5568)$ state. In other words, the quark content of $X_{c}=[s u][\bar{c} \bar{d}]$ was obtained from $X_{b}=[s u][\overline{b d}]$ by $b \rightarrow c$ replacement. Due to differences in the charges of $b$ and $c$ quarks the partner state $X_{c}$ does not bear the same charge as $X_{b}$. This conclusion is true in the case of $Z_{\bar{c} s}$, as well. If the state $Z_{\bar{c} s}$ bears the charge $-2|e|$, its $b$-partner $Z_{\bar{b} s}$ has $-|e|$. In general, there do not exist doubly charged tetraquarks composed of $b$ and three different light quarks. The genuine doubly charged tetraquarks with $b$ belong to a subclass of open charm-bottom particles and should contain also $c$-quark. For example, the state $Z_{b \bar{c}}=[b s][\overline{u c}]$ has the charge $-2|e|$.

In the present work we are going to concentrate on features of doubly charged charm-strange tetraquarks $Z_{\bar{c} s}$ with spinparity $J^{P}=0^{+}, 0^{-}$and $1^{+}$, and calculate their masses, current couplings and decay widths. To this end, we use QCD two-point sum rule approach by including into analysis quark, gluon and mixed vacuum condensates up to eight dimensions, and evaluate their spectroscopic parameters. Obtained results are employed to reveal kinematically allowed decay channels of the tetraquarks $Z_{\bar{c} s}$. They also enter as input parameters to expressions of the corresponding decay widths. We calculate the width of decay channels $Z_{\bar{c} s} \rightarrow D_{s} \pi, D K$, and $D_{s 1}(2460) \pi$ (for $J^{P}=0^{+}$),
$Z_{\bar{c} s} \rightarrow D_{s}^{*} \pi, D^{*} K$ and $D_{s 0}^{*}(2317) \pi$ (for $J^{P}=0^{-}$), as well as $Z_{\bar{c} s} \rightarrow D_{s}^{*} \pi, D^{*} K$ and $D_{s 1}(2460) \pi$ (in the case of $J^{P}=1^{+}$). For these purposes, we analyze vertices of the tetraquarks $Z_{\bar{c} s}$ with the conventional mesons, and evaluate the corresponding strong couplings using QCD sum rules on the light-cone. The QCD light-cone sum rule method is one of the powerful nonperturbative tools to explore parameters of the conventional hadrons [40]. In the case of vertices built of a tetraquark and two conventional mesons the standard methods of the light-cone sum rules should be supplemented by a technique of an approach known as the "soft-meson" approximation [41,42]. For investigation of the exotic states the light-cone sum rules method was adapted in Ref. [43], and successfully applied for analysis of various tetraquarks' decays [44-47].

This article is organized in the following manner. In Sect. 2 we calculate the masses and current couplings of the doubly charged scalar, pseudoscalar and axial-vector charmstrange tetraquarks $Z_{\bar{c} s}=[s d][\overline{u c}]$ by treating them as diquark-antidiquark systems. In Sect. 3 we consider the decays of the doubly charged scalar tetraquark to $D_{s} \pi, D K$ and $D_{s 1}(2460) \pi$ final states. Section 4 is devoted to decay channels of the pseudoscalar and axial-vector tetraquarks. Here we compute width of their decays to $D_{s}^{*} \pi, D^{*} K$ and $D_{s 0}^{*}(2317) \pi$ (for $0^{-}$state), and to $D_{s}^{*} \pi, D^{*} K$ and $D_{s 1}(2460) \pi$ (for $1^{+}$state). Section 5 is reserved for our concluding remarks.

\section{Spectroscopic parameters of the scalar, pseudoscalar and axial vector tetraquarks $Z_{\bar{c} s}$}

In this section we calculate the mass and current coupling of the $Z_{\bar{c} s}=[s d][\overline{u c}]$ tetraquarks with the quantum numbers $J^{P}=0^{+}, 0^{-}$and $1^{+}$by treating them as diquak-antidiquark systems. In order to simplify the expressions we introduce the notations: in what follows the scalar tetraquark $Z_{\bar{c} s}$ will be denoted as $Z_{S}$, whereas for the pseudoscalar and axial-vector ones we will utilize $Z_{P S}$ and $Z_{A V}$, respectively.

The scalar tetraquarks within the context of two-point sum rule approach can be explored using interpolating currents of $C \gamma_{5} \otimes \gamma_{5} C$ or $C \gamma_{\mu} \otimes \gamma^{\mu} C$ types, where $C$ is the charge conjugation operator. In the present work we restrict ourselves by the simplest case and employ the current

$J=s_{a}^{T} C \gamma_{5} d_{b}\left(\bar{u}_{a} \gamma_{5} C \bar{c}_{b}^{T}-\bar{u}_{b} \gamma_{5} C \bar{c}_{a}^{T}\right)$.

To study the pseudoscalar and axial-vector tetraquarks $Z_{P S}$ and $Z_{A V}$ we utilize $C \gamma_{\mu} \otimes \gamma_{5} C$ type interpolating current

$J_{\mu}=s_{a}^{T} C \gamma_{\mu} d_{b}\left(\bar{u}_{a} \gamma_{5} C \bar{c}_{b}^{T}-\bar{u}_{b} \gamma_{5} C \bar{c}_{a}^{T}\right)$. 
Then the correlation functions $\Pi(p)$ and $\Pi_{\mu \nu}(p)$ necessary for the sum rule computations take the forms

$\Pi(p)=i \int d^{4} x e^{i p x}\left\langle 0\left|\mathcal{T}\left\{J(x) J^{\dagger}(0)\right\}\right| 0\right\rangle$,

and

$\Pi_{\mu \nu}(p)=i \int d^{4} x e^{i p x}\left\langle 0\left|\mathcal{T}\left\{J_{\mu}(x) J_{v}^{\dagger}(0)\right\}\right| 0\right\rangle$.

The current $J_{\mu}$ couples to both the pseudoscalar $0^{-}$and axialvector $1^{+}$states, therefore the function $\Pi_{\mu \nu}(p)$ can be used to calculate parameters of the $Z_{P S}$ and $Z_{A V}$ tetraquarks.

We start our analysis from calculations of the scalar state's spectroscopic parameters. In accordance with QCD sum rule method the correlator given by Eq. (3) should be expressed in terms of physical parameters of the $Z_{S}$ state. In the case under analysis $\Pi(p)$ takes simple form and is defined by the equality

$\Pi^{\text {Phys }}(p)=\frac{\left\langle 0|J| Z_{S}(p)\right\rangle\left\langle Z_{S}(p)\left|J^{\dagger}\right| 0\right\rangle}{m_{Z_{S}}^{2}-p^{2}}+\cdots$,

where $m_{Z_{S}}$ is the mass of the $Z_{S}$ state, and dots stand for contributions of the higher resonances and continuum states. In order to simplify $\Pi^{\text {Phys }}(p)$ we introduce the matrix element

$\left\langle 0|J| Z_{S}(p)\right\rangle=f_{Z_{S}} m_{Z_{S}}$,

where $m_{Z_{S}}$ and $f_{Z_{S}}$ are the mass and current coupling of $Z_{S}(p)$. Then, for the correlation function we obtain

$\Pi^{\text {Phys }}(p)=\frac{m_{Z_{S}}^{2} f_{Z_{S}}^{2}}{m_{Z_{S}}^{2}-p^{2}}+\cdots$.

The Borel transformation applied to $\Pi^{\text {Phys }}(p)$ yields

$\mathcal{B} \Pi^{\text {Phys }}(p)=m_{Z_{S}}^{2} f_{Z_{S}}^{2} e^{-m_{Z_{S}}^{2} / M^{2}}+\cdots$,

where $M^{2}$ is the Borel parameter.

The same correlation function $\Pi(p)$ calculated in terms of the quark-gluon degrees of freedom reads

$$
\begin{aligned}
& \Pi^{\mathrm{QCD}}(p)=i \int d^{4} x e^{i p x}\left\{\operatorname{Tr}\left[\gamma_{5} \widetilde{S}_{c}^{b^{\prime} b}(-x) \gamma_{5} S_{u}^{a^{\prime} a}(-x)\right]\right. \\
& \quad \times \operatorname{Tr}\left[S_{s}^{a a^{\prime}}(x) \gamma_{5} \widetilde{S}_{d}^{b b^{\prime}}(x) \gamma_{5}\right]-\operatorname{Tr}\left[\gamma_{5} \widetilde{S}_{c}^{a^{\prime} b}(-x)\right. \\
& \left.\quad \times \gamma_{5} S_{u}^{b^{\prime} a}(-x)\right] \operatorname{Tr}\left[S_{s}^{a a^{\prime}}(x) \gamma_{5} \widetilde{S}_{d}^{b b^{\prime}}(x) \gamma_{5}\right] \\
& \quad-\operatorname{Tr}\left[\gamma_{5} \widetilde{S}_{c}^{b^{\prime} a}(-x) \gamma_{5} S_{u}^{a^{\prime} b}(-x)\right] \operatorname{Tr}\left[S_{s}^{a a^{\prime}}(x) \gamma_{5} \widetilde{S}_{d}^{b b^{\prime}}(x) \gamma_{5}\right] \\
& \left.\quad+\operatorname{Tr}\left[\gamma_{5} \widetilde{S}_{c}^{a^{\prime} a}(-x) \gamma_{5} S_{u}^{b^{\prime} b}(-x)\right] \operatorname{Tr}\left[S_{s}^{a a^{\prime}}(x) \gamma_{5} \widetilde{S}_{d}^{b^{\prime} b}(x) \gamma_{5}\right]\right\} .
\end{aligned}
$$

Here we use the short-hand notation

$\tilde{S}_{q(c)}^{a b}(x)=C S_{q(c)}^{T a b}(x) C$,

with $S_{q}(x)$ and $S_{c}(x)$ being the $q=u, d$ and $c$-quark propagators, respectively.
The QCD sum rules to evaluate $m_{Z_{S}}$ and $f_{Z_{S}}$ can be obtained by choosing the same Lorentz structures in both of $\Pi^{\mathrm{Phys}}(p)$ and $\Pi^{\mathrm{QCD}}(p)$, and equating the relevant invariant amplitudes. In the case under investigation the only Lorentz structure which exists in $\Pi(p)$ is one $\sim I$. For calculation of the mass and coupling it is convenient to employ the twopoint spectral density $\rho_{0}^{\mathrm{QCD}}(s)$. In terms of $\rho_{0}^{\mathrm{QCD}}(s)$ the invariant amplitude $\Pi^{\mathrm{QCD}}\left(p^{2}\right)$ can be written down as the dispersion integral

$\Pi^{\mathrm{QCD}}\left(p^{2}\right)=\int_{\left(m_{c}+m_{s}\right)^{2}}^{\infty} \frac{\rho_{0}^{\mathrm{QCD}}(s)}{s-p^{2}} d s+\cdots$.

By applying the Borel transformation to $\Pi^{\mathrm{QCD}}\left(p^{2}\right)$, equating the obtained expression with $\mathcal{B} \Pi^{\mathrm{Phys}}\left(p^{2}\right)$, and subtracting the contribution due to higher excited and continuum states we find the final sum rules. For the mass of the $Z_{S}$ state it is given by the formula

$m_{Z_{S}}^{2}=\frac{\int_{\left(m_{c}+m_{s}\right)^{2}}^{s_{0}} d s s \rho_{0}^{\mathrm{QCD}}(s) e^{-s / M^{2}}}{\int_{\left(m_{c}+m_{s}\right)^{2}}^{s^{2}} d s \rho_{0}^{\mathrm{QCD}}(s) e^{-s / M^{2}}}$,

whereas for the current coupling $f_{Z_{S}}$ we get

$f_{Z_{S}}^{2}=\frac{1}{m_{Z_{S}}^{2}} \int_{\left(m_{c}+m_{s}\right)^{2}}^{s_{0}} d s e^{\left(m_{Z_{S}}^{2}-s\right) / M^{2}} \rho_{0}^{\mathrm{QCD}}(s)$.

In Eqs. (12) and (13) $s_{0}$ is the continuum threshold parameter which separates contributions stemming from the groundstate and ones due to higher resonances and continuum states. The $M^{2}$ and $s_{0}$ are two important auxiliary parameters of sum rule computations choices of which should meet some requirements which will be shortly explained below.

In the case of the current $J_{\mu}$ the correlation function $\Pi_{\mu \nu}^{\text {Phys }}(p)$ derived using the physical parameters of tetraquarks contains two terms. In fact, the current $J_{\mu}$ couples to the pseudoscalar and axial-vector tetraquarks, therefore after inserting into Eq. (4) full set of states and integrating over $x$ we get expression containing contributions of the ground state pseudoscalar and axial-vector particles, i.e.

$$
\begin{aligned}
\Pi_{\mu \nu}^{\text {Phys }}(p)= & \frac{\left\langle 0\left|J_{\mu}\right| Z_{A V}(p)\right\rangle\left\langle Z_{A V}(p)\left|J_{v}^{\dagger}\right| 0\right\rangle}{m_{Z_{A V}}^{2}-p^{2}} \\
& +\frac{\left\langle 0\left|J_{\mu}\right| Z_{P S}(p)\right\rangle\left\langle Z_{P S}(p)\left|J_{v}^{\dagger}\right| 0\right\rangle}{m_{Z_{P S}}^{2}-p^{2}}+\cdots,
\end{aligned}
$$

with $m_{Z_{P S}}$ and $m_{Z_{A V}}$ being the mass of the pseudoscalar and axial-vector states, respectively. Here again by dots we denote contributions coming from higher excitations and continuum states in both the pseudoscalar and vector channels. In general one may consider only one of these terms, and compute parameters of the chosen particle with $J^{P}=0^{-}$ or $1^{+}$. In the present work we are interested in both of these 
particles, therefore keep explicitly two terms in $\Pi_{\mu \nu}^{\text {Phys }}(p)$, and use different structures to derive two sets of sum rules.

Further simplification of $\Pi_{\mu \nu}^{\text {Phys }}(p)$ can be achieved by expressing the relevant matrix elements in terms of the masses $m_{Z_{P S}}, m_{Z_{A V}}$ and current couplings $f_{Z_{A V}}, f_{Z_{P S}}$

$$
\begin{aligned}
\left\langle 0\left|J_{\mu}\right| Z_{A V}(p)\right\rangle & =f_{Z_{A V}} m_{Z_{A V}} \varepsilon_{\mu}(p), \\
\left\langle 0\left|J_{\mu}\right| Z_{P S}(p)\right\rangle & =f_{Z_{P S}} m_{Z_{P S}} p_{\mu} .
\end{aligned}
$$

Then it is easy to show that

$$
\begin{aligned}
\Pi_{\mu \nu}^{\text {Phys }}(p)= & \frac{m_{Z_{A V}}^{2} f_{Z_{A V}}^{2}}{m_{Z_{A V}}^{2}-p^{2}}\left(-g_{\mu \nu}+\frac{p_{\mu} p_{v}}{p^{2}}\right) \\
& +\frac{m_{Z_{P S}}^{2} f_{Z_{P S}}^{2}}{m_{Z_{P S}}^{2}-p^{2}} p_{\mu} p_{v}+\cdots
\end{aligned}
$$

In order to obtain the function $\Pi_{\mu \nu}^{\mathrm{QCD}}(p)$ we substitute the interpolating current $J_{\mu}$ from Eq. (2) into Eq. (4), and contract the quark fields. As a result, for $\Pi_{\mu \nu}^{\mathrm{QCD}}(p)$ we get:

$$
\begin{aligned}
& \Pi_{\mu \nu}^{\mathrm{QCD}}(p)=i \int d^{4} x e^{i p x}\left\{\operatorname{Tr}\left[\gamma_{5} \widetilde{S}_{c}^{a^{\prime} b}(-x) \gamma_{5} S_{u}^{b^{\prime} a}(-x)\right]\right. \\
& \quad \times \operatorname{Tr}\left[S_{s}^{a a^{\prime}}(x) \gamma_{\nu} \widetilde{S}_{d}^{b b^{\prime}}(x) \gamma_{\mu}\right]+\operatorname{Tr}\left[\gamma_{5} \widetilde{S}_{c}^{b^{\prime} a}(-x)\right. \\
& \left.\quad \times \gamma_{5} S_{u}^{a^{\prime} b}(-x)\right] \operatorname{Tr}\left[S_{s}^{a a^{\prime}}(x) \gamma_{\nu} \widetilde{S}_{d}^{b b^{\prime}}(x) \gamma_{\mu}\right] \\
& -\operatorname{Tr}\left[\gamma_{5} \widetilde{S}_{c}^{b^{\prime} b}(-x) \gamma_{5} S_{u}^{a^{\prime} a}(-x)\right] \operatorname{Tr}\left[S_{s}^{a a^{\prime}}(x) \gamma_{v} \widetilde{S}_{d}^{b b^{\prime}}(x) \gamma_{\mu}\right] \\
& \left.-\operatorname{Tr}\left[\gamma_{5} \widetilde{S}_{c}^{a^{\prime} a}(-x) \gamma_{5} S_{u}^{b^{\prime} b}(-x)\right] \operatorname{Tr}\left[S_{s}^{a a^{\prime}}(x) \gamma_{v} \widetilde{S}_{d}^{b b^{\prime}}(x) \gamma_{\mu}\right]\right\} .
\end{aligned}
$$

The correlation function $\Pi_{\mu \nu}^{\mathrm{QCD}}(p)$ has the following Lorentz structures

$$
\begin{aligned}
\Pi_{\mu \nu}^{\mathrm{QCD}}(p)= & \Pi_{A V}^{\mathrm{QCD}}\left(p^{2}\right)\left(-g_{\mu \nu}+\frac{p_{\mu} p_{v}}{p^{2}}\right) \\
& +\frac{p_{\mu} p_{v}}{p^{2}} \Pi_{P S}^{\mathrm{QCD}}\left(p^{2}\right),
\end{aligned}
$$

where $\Pi_{A V}^{\mathrm{QCD}}\left(p^{2}\right)$ and $\Pi_{P S}^{\mathrm{QCD}}\left(p^{2}\right)$ are invariant amplitudes corresponding to the axial-vector and pseudoscalar tetraquarks, respectively. Equating the structures $\sim g_{\mu \nu}$ in Eqs. (15) and (17), and performing the Borel transformation it is possible we derive the sum rules for parameters of the axialvector tetraquark: They are given by Eqs. (12) and (13) but with $\rho_{0}^{\mathrm{QCD}}(s)$ replaced by $\rho_{V}^{\mathrm{QCD}}(s)$.

The sum rules for the pseudoscalar state are found by computing $p^{\mu} \Pi_{\mu \nu}^{\text {Phys }}(p)$ and $p^{\mu} \Pi_{\mu \nu}^{\mathrm{QCD}}(p)$, and matching obtained expressions, which consist of terms with parameters of the pseudoscalar tetraquark. Then for the mass of the pseudoscalar state we again find the sum rule (12), but $\rho_{0}^{\mathrm{QCD}}(s) \rightarrow \rho_{S}^{\mathrm{QCD}}(s)$, whereas the coupling $f_{Z_{P S}}$ is determined by the following expression

$f_{Z_{P S}}^{2}=\frac{1}{m_{Z_{P S}}^{4}} \int_{\left(m_{c}+m_{s}\right)^{2}}^{s_{0}} d s e^{\left(m_{Z_{P S}}^{2}-s\right) / M^{2}} \rho_{S}^{\mathrm{QCD}}(s)$.
In the present work we calculate the two-point spectral densities $\rho_{0}^{\mathrm{QCD}}(s), \rho_{V}^{\mathrm{QCD}}(s)$ and $\rho_{S}^{\mathrm{QCD}}(s)$ by taking into account quark, gluon and mixed vacuum condensates up to eight dimensions.

The sum rules (12), (13) and (18) depend on the masses of $c$ and $s$-quarks, and vacuum expectations of quark, gluon and mixed operators, which are presented below:

$$
\begin{aligned}
& m_{c}=(1.27 \pm 0.03) \mathrm{GeV}, \quad m_{s}=96_{-4}^{+8} \mathrm{MeV} \\
& \langle\bar{q} q\rangle=-(0.24 \pm 0.01)^{3} \mathrm{GeV}^{3},\langle\bar{s} s\rangle=0.8\langle\bar{q} q\rangle \\
& m_{0}^{2}=(0.8 \pm 0.1) \mathrm{GeV}^{2},\left\langle\bar{q} g_{s} \sigma G q\right\rangle=m_{0}^{2}\langle\bar{q} q\rangle \\
& \left\langle\bar{s} g_{s} \sigma G s\right\rangle=m_{0}^{2}\langle\bar{s} s\rangle \\
& \left\langle\frac{\alpha_{s} G^{2}}{\pi}\right\rangle=(0.012 \pm 0.004) \mathrm{GeV}^{4} \\
& \left\langle g_{s}^{3} G^{3}\right\rangle=(0.57 \pm 0.29) \mathrm{GeV}^{6}
\end{aligned}
$$

For condensates we use their standard values, whereas the masses of the quarks are borrowed from Ref. [48]. In the chiral limit adopted in the present work $m_{u}=m_{d}=0$.

The sum rules contain also, as it has been just noted above, the auxiliary parameters $M^{2}$ and $s_{0}$. It is clear, that physical quantities evaluated from the sum rules should not depend on the Borel parameter and continuum threshold, but in real calculations one can only reduce their effect to a minimum. In fixing of working regions for $M^{2}$ and $s_{0}$ some conditions should be obeyed. Thus, we fix the upper bound $M_{\max }^{2}$ of the window $M^{2} \in\left[M_{\min }^{2}, M_{\max }^{2}\right]$ for the Borel parameter by requiring fulfilment of the following constraint

$\mathrm{PC}=\frac{\Pi^{\mathrm{QCD}}\left(M_{\max }^{2}, s_{0}\right)}{\Pi^{\mathrm{QCD}}\left(M_{\max }^{2}, \infty\right)}>0.13$,

where $\Pi^{\mathrm{QCD}}\left(M^{2}, s_{0}\right)=\mathcal{B} \Pi^{\mathrm{QCD}}\left(p^{2}\right)$ is the Borel transform of the invariant amplitude after the continuum subtraction. Minimal limit for PC chosen as $\sim 0.1$ is smaller than in the case of the conventional mesons, but is typical for multiquark systems. The lower limit of the same region $M_{\mathrm{min}}^{2}$ is deduced from convergence of the operator product expansion. By quantifying this condition we require that contribution of the last term in OPE should not exceed $5 \%$, i. e.

$$
\frac{\Pi^{\mathrm{QCD}(\operatorname{Dim} 8)}\left(M_{\min }^{2}, \infty\right)}{\Pi^{\mathrm{QCD}}\left(M_{\min }^{2}, \infty\right)}<0.05,
$$

has to be obeyed. Another condition for the lower limit is exceeding of the perturbative contribution the nonperturbative one. In the present work we apply the following criterion: at the lower bound of $M^{2}$ the perturbative contribution has to constitute $\geq 60 \%$ part of the full result.

Analysis of the sum rules for the $Z_{S}$ state enable us to fix the Borel and continuum threshold parameters within the limits:

$M^{2} \in[2.5-3.5] \mathrm{GeV}^{2}, s_{0} \in[8-10] \mathrm{GeV}^{2}$. 
In these regions the pole contribution defined by Eq. (20) is $\mathrm{PC}>0.14$. At the same time, contribution coming from the pole term at $M_{\text {min }}^{2}$ constitutes $\sim 65 \%$ and at $M_{\min }^{2}$ approximately $60 \%$ of the sum rule (12) used to evaluate the mass of $Z_{S}$ state. The convergence of OPE expansion in these regions is also satisfied. Thus, contribution of the Dim8 term in OPE

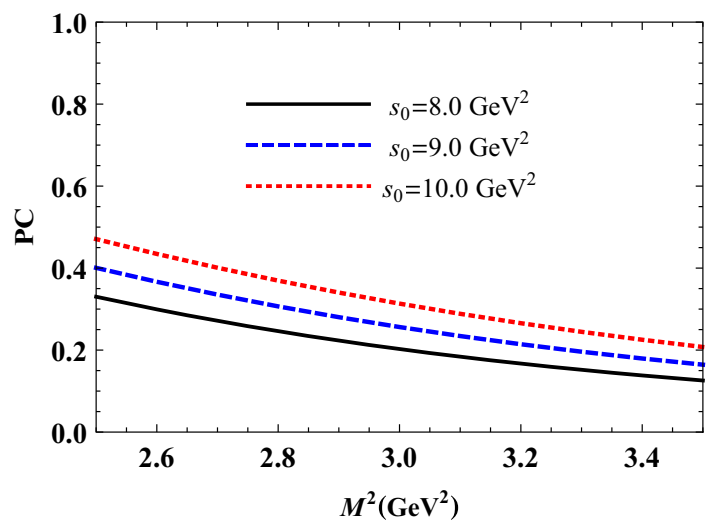

Fig. 1 The pole contribution in the case of the scalar tetraquark vs the Borel parameter $M^{2}$ at different $s_{0}$

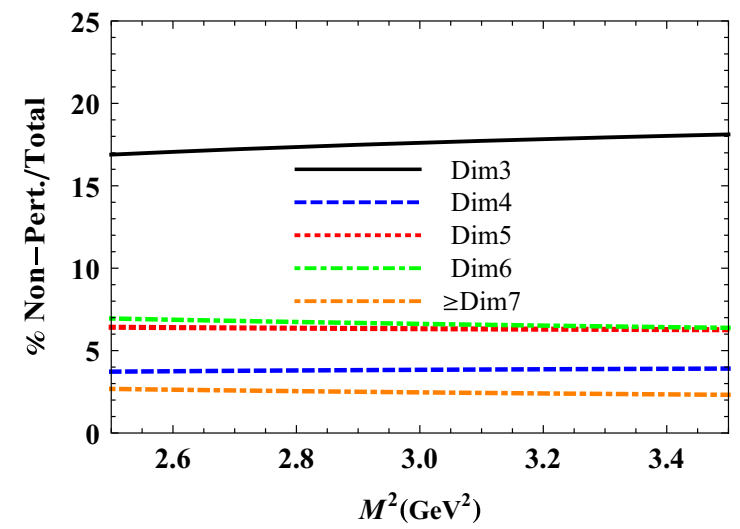

does not exceed 3\%. All these features are seen in Figs. 1, 2 and 3 , where we plot the pole contribution, contributions due to different nonperturbative terms, and the perturbative and total nonperturbative components of $\Pi^{\mathrm{QCD}}\left(M^{2}, s_{0}\right)$ to demonstrate that in the regions for $M^{2}$ and $s_{0}$ given by Eq. (22) constraints imposed on $\Pi^{\mathrm{QCD}}\left(M^{2}, s_{0}\right)$ are fulfilled.

From obtained sum rules for the mass and current coupling of $Z_{S}$ state we find

$$
\begin{aligned}
m_{Z_{S}} & =2628_{-153}^{+166} \mathrm{MeV} \\
f_{Z_{S}} & =\left(0.21_{-0.05}^{+0.06}\right) \times 10^{-2} \mathrm{GeV}^{4}
\end{aligned}
$$

In Figs. 4 and 5, $m_{Z_{S}}$ and $f_{Z_{S}}$ are depicted as functions of $M^{2}$ and $s_{0}$. It is seen that while effects of varying of these parameters on the mass $m_{Z_{S}}$ are small, dependence of the current coupling $f_{Z_{S}}$ on chosen values of the continuum threshold parameter is noticeable. These effects together with uncertainties of the input parameters generate the theoretical errors in the sum rule calculations, which are their unavoidable feature and may reach $30 \%$ of the central values.

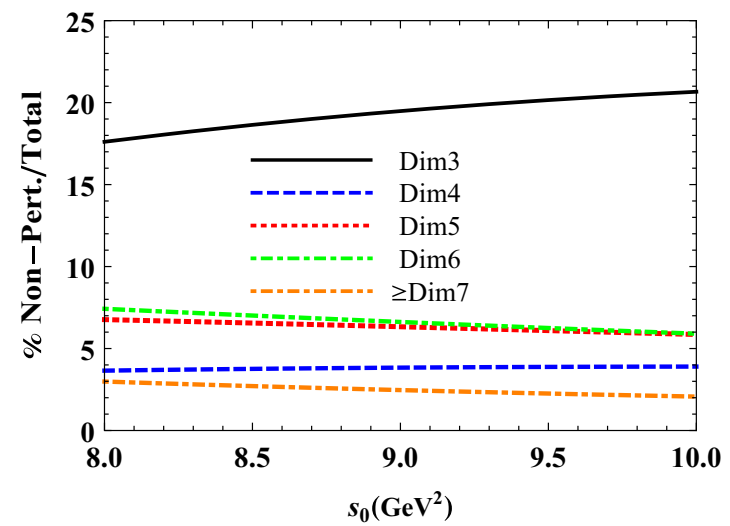

Fig. 2 Contributions due to different nonperturbative terms in the case of $Z_{S}$ as functions of $M^{2}$ (left panel), and $s_{0}$ (right panel)
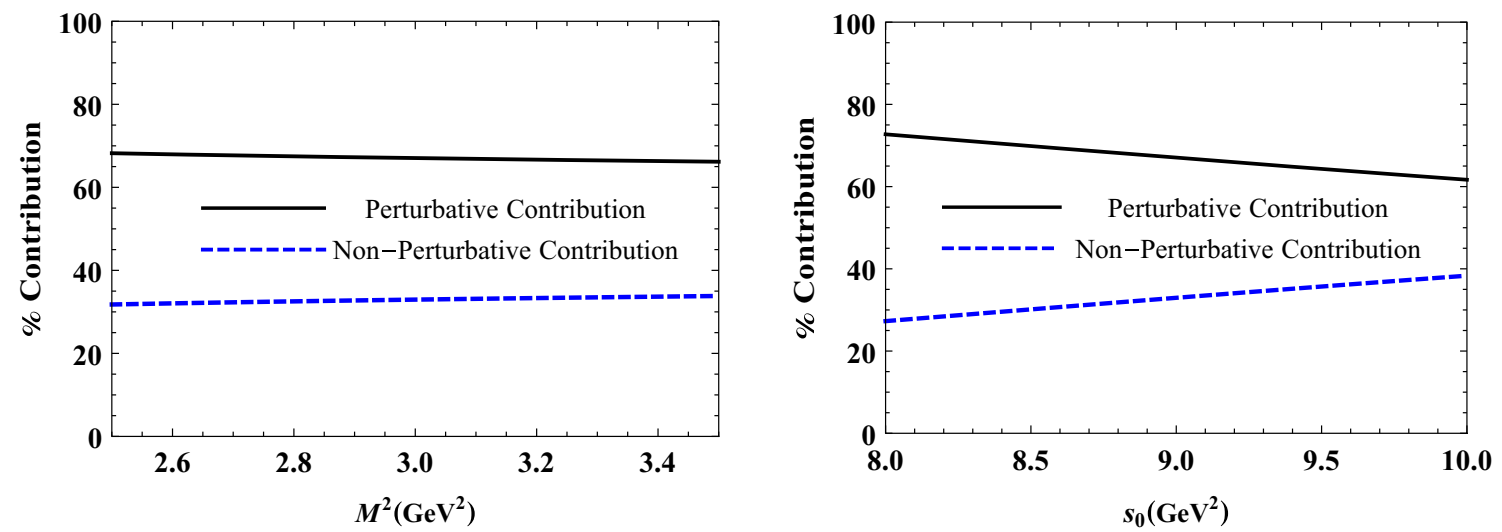

Fig. 3 The perturbative and nonperturbative contributions to $\Pi^{\mathrm{QCD}}\left(M^{2}, s_{0}\right)$ of the scalar particle. Left: as functions of $M^{2}$ at central value $s_{0}=9 \mathrm{GeV}^{2}$, right: as functions of $s_{0}$ at $M^{2}=3 \mathrm{GeV}^{2}$ 

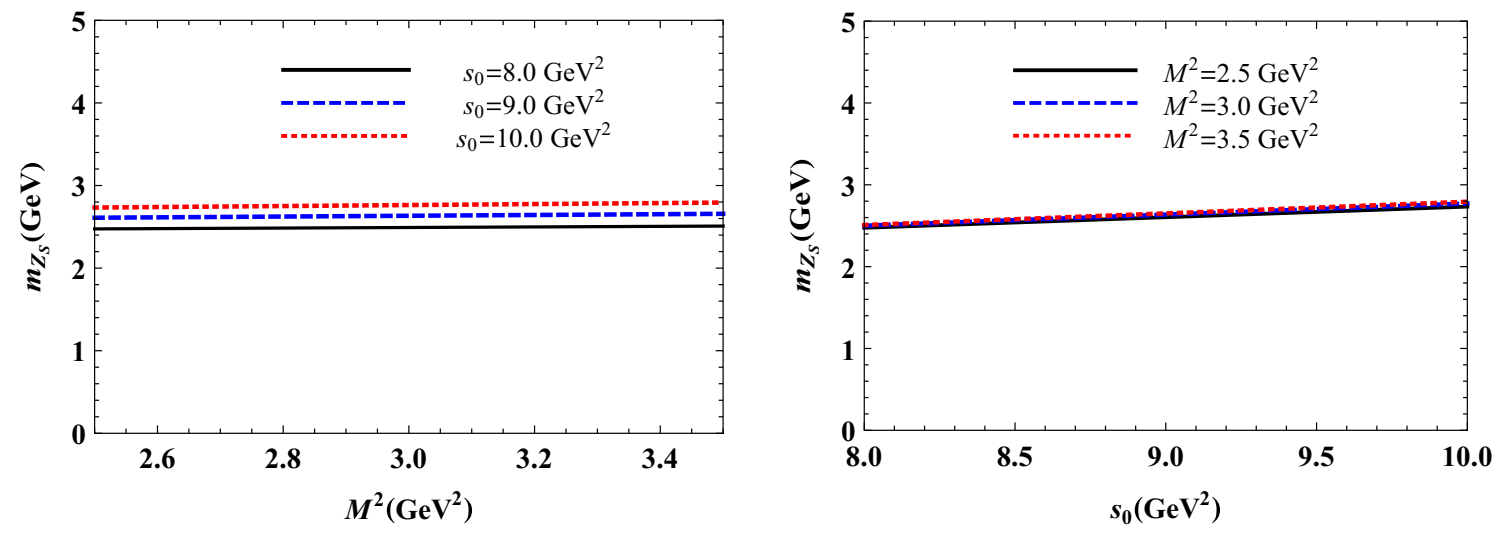

Fig. 4 The mass of the $Z_{S}$ state as a function of the Borel parameter $M^{2}$ at fixed values of $s_{0}$ (left panel), and as a function of the continuum threshold $s_{0}$ at fixed $M^{2}$ (right panel)
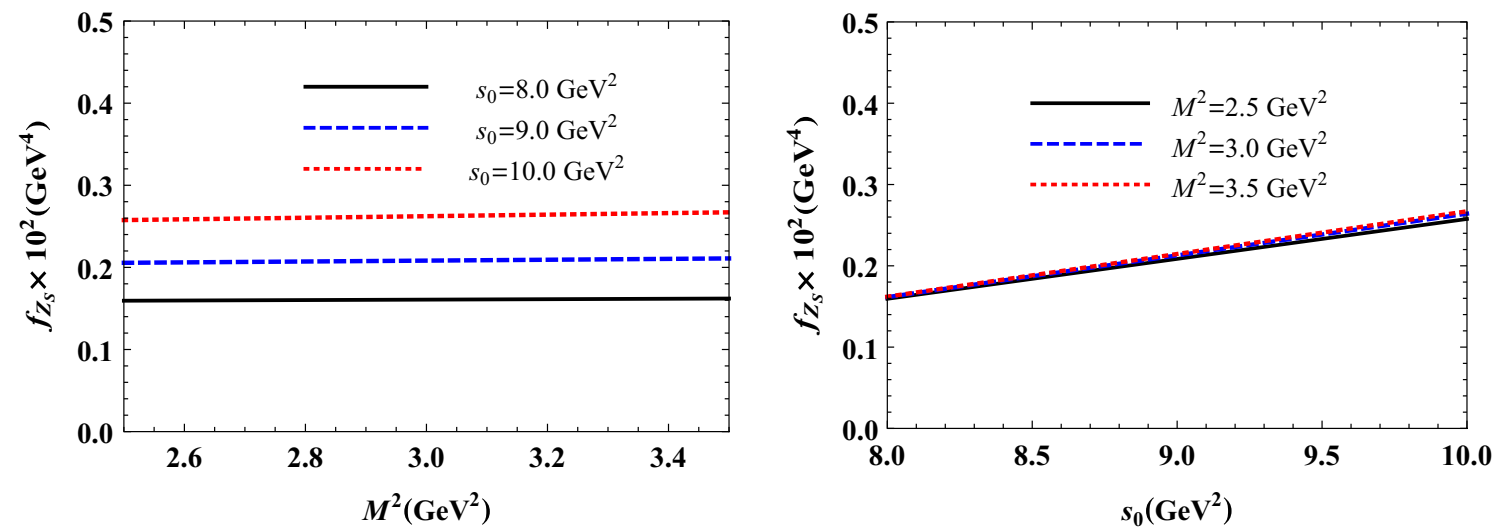

Fig. 5 The dependence of the current coupling $f_{Z_{S}}$ of the scalar $Z_{S}$ tetraquark on the Borel parameter at chosen values of $s_{0}$ (left panel), and on the continuum threshold parameter $s_{0}$ at fixed $M^{2}$ (right panel)

The analogous studies can be carried out for the pseudoscalar and axial-vector tetraquarks. From performed analysis we conclude that regions

$M^{2} \in[2.5-3.5] \mathrm{GeV}^{2}, s_{0} \in[9.5-11.5] \mathrm{GeV}^{2}$

can be used to evaluate the spectroscopic parameters of the pseudoscalar and axial-vector tetraquarks, as well. Results of computations for the axial-vector state are depicted in Figs. 6,7 and 8 , which confirm our conclusions. The similar results are also valid for the pseudoscalar tetraquark $Z_{P S}$.

In Figs. 9 and 10 we plot dependence of the axial-vector tetraquark's mass and current coupling on $M^{2}$ and $s_{0}$. As is seen, estimations made for theoretical errors in the case of $Z_{S}$ are valid for the $Z_{A V}$ state, as well.

Our results for the masses and current couplings of $J^{P}=$ $0^{+}, 0^{-}$and $J^{P}=1^{+}$charm-strange tetraquarks are collected in Table 1. The working ranges for the parameters $M^{2}$ and $s_{0}$, and errors of the calculations are also presented in Table 1.

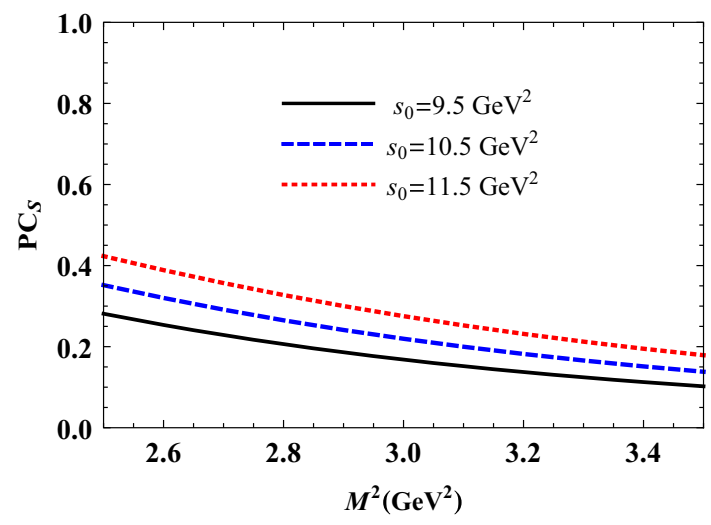

Fig. 6 The pole contribution in the case of the axial-vector tetraquark as a function of the Borel parameter $M^{2}$

\section{Decay channels of the scalar tetraquark $Z_{S}$}

In this section we calculate the width of processes $Z_{S} \rightarrow$ $D_{s} \pi, Z_{S} \rightarrow D K$ and $Z_{S} \rightarrow D_{s 1}(2460) \pi$, which in the light of the result obtained for $m_{Z_{S}}$ are kinematically allowed 

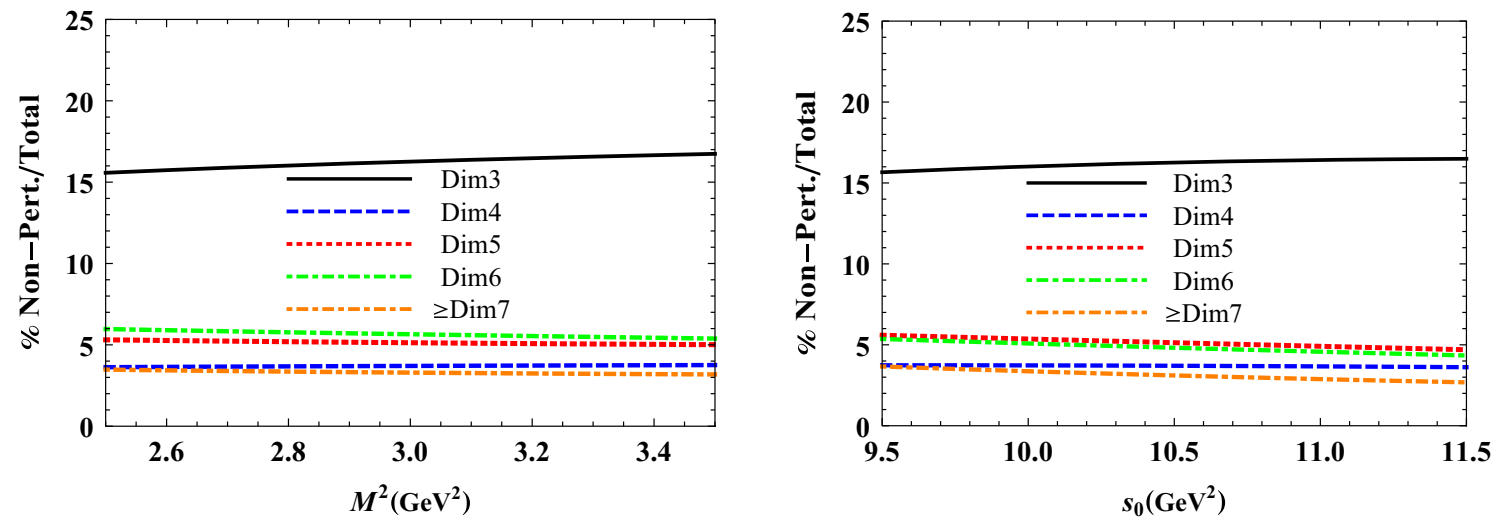

Fig. 7 Nonperturbative contributions to $\Pi^{\mathrm{QCD}}\left(M^{2}, s_{0}\right)_{A V}$ as functions of $M^{2}$ (left panel), and $s_{0}$ (right panel)
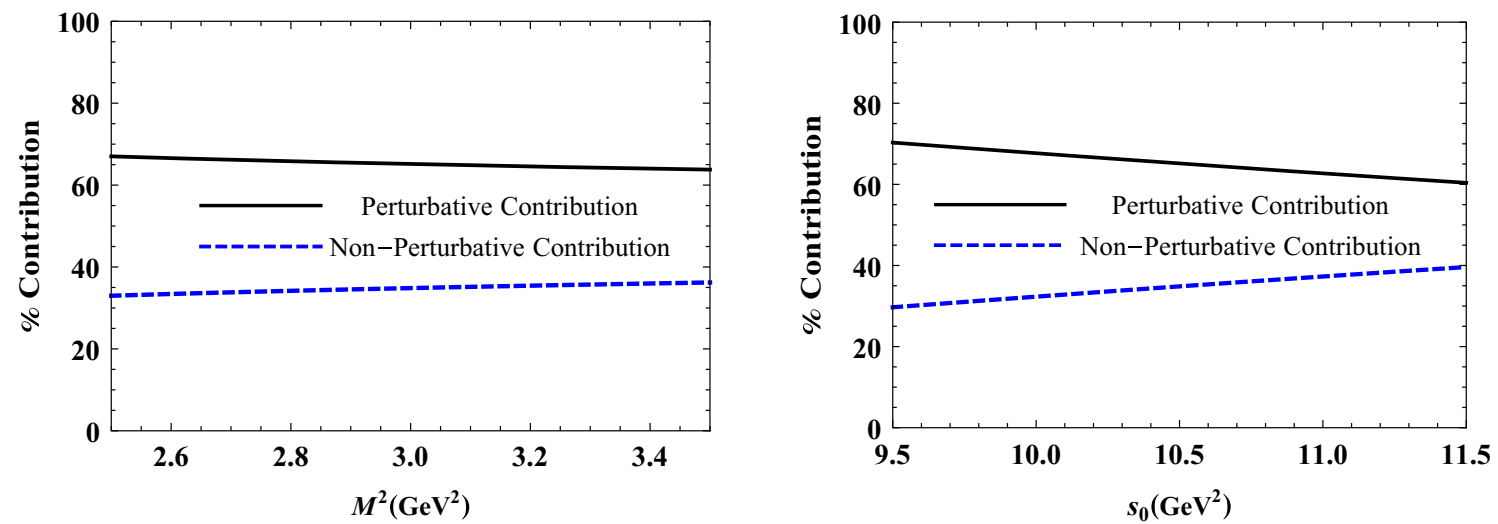

Fig. 8 The perturbative and nonperturbative components of $\Pi^{\mathrm{QCD}}\left(M^{2}, s_{0}\right)_{A V}$. Left: as functions of $M^{2}$ at $s_{0}=10.5 \mathrm{GeV}^{2}$, right: as functions of $s_{0}$ at the central value of the Borel parameter $M^{2}=3 \mathrm{GeV}^{2}$
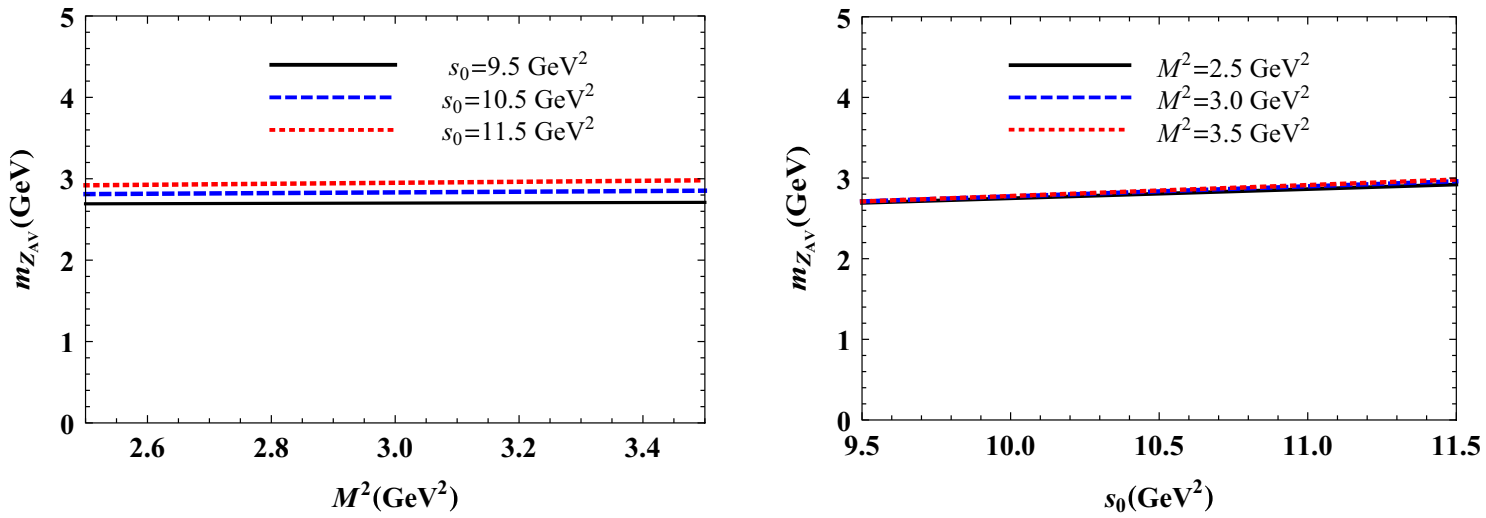

Fig. 9 The mass of the $Z_{A V}$ state vs Borel parameter $M^{2}$ at fixed values of $s_{0}$ (left panel), and vs continuum threshold $s_{0}$ at fixed values of $M^{2}$ (right panel)

decay channels of the scalar tetraquark. It is evident that in all these channels the final mesons are particles with negative charges. For simplicity of expressions throughout this paper we do not show explicitly charges of the final mesons. Let us note that first two processes are $S$-wave decay modes, whereas the last one is $P$-wave decay.
In order to evaluate the width of these decays we have to calculate the strong couplings corresponding to the vertices $Z_{S} D_{S} \pi, Z_{S} D K$ and $Z_{S} D_{s 1}(2460) \pi$. This task can be fulfilled by analysis of corresponding correlation functions and calculating them using light-cone sum rule method. To calculate the strong coupling $g_{Z_{S} D_{S} \pi}$ and width of the decay $Z_{S} \rightarrow D_{s} \pi$ we consider the correlator 

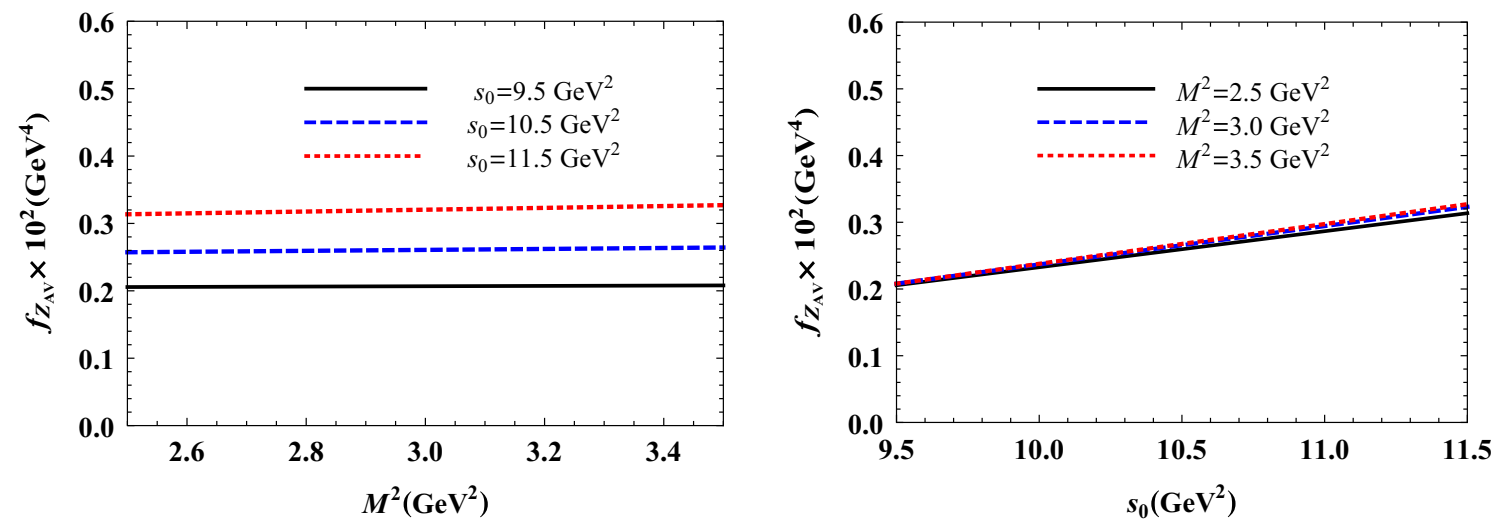

Fig. 10 The current coupling $f_{Z_{A V}}$ of the $Z_{A V}$ state vs Borel parameter $M^{2}$ at chosen values of $s_{0}$ (left panel), and vs $s_{0}$ at fixed values of $M^{2}$ (right panel)

Table 1 The masses and current couplings of the $Z_{S}$, $Z_{P S}$ and $Z_{A V}$ tetraquarks

\begin{tabular}{llll}
\hline$Z$ & $Z_{S}$ & $Z_{P S}$ & $Z_{A V}$ \\
\hline$M^{2}\left(\mathrm{GeV}^{2}\right)$ & $2.5-3.5$ & $2.5-3.5$ & $2.5-3.5$ \\
$s_{0}\left(\mathrm{GeV}^{2}\right)$ & $8-10$ & $9.5-11.5$ & $9.5-11.5$ \\
$m_{Z}(\mathrm{MeV})$ & $2628_{-153}^{+166}$ & $2719_{-156}^{+144}$ & $2826_{-157}^{+134}$ \\
$f_{Z} \times 10^{3}$ & $2.1_{-0.5}^{+0.6}\left(\mathrm{GeV}^{4}\right)$ & $0.83_{-0.11}^{+0.09}\left(\mathrm{GeV}^{3}\right)$ & $2.6_{-0.7}^{+0.6}\left(\mathrm{GeV}^{4}\right)$ \\
\hline
\end{tabular}

$\Pi(p, q)=i \int d^{4} x e^{i p x}\left\langle\pi(q)\left|\mathcal{T}\left\{J^{D_{s}}(x) J^{\dagger}(0)\right\}\right| 0\right\rangle$,

where

$J^{D_{s}}(x)=\bar{c}_{i}(x) \gamma_{5} s_{i}(x)$,

is the interpolating current of the pseudoscalar meson $D_{s}$.

The correlation function $\Pi(p, q)$ in terms of the physical parameters of the involved particles is equal to

$$
\begin{aligned}
\Pi^{\text {Phys }}(p, q)= & \frac{\left\langle 0\left|J^{D_{s}}\right| D_{s}(p)\right\rangle}{p^{2}-m_{D_{s}}^{2}}\left\langle D_{s}(p) \pi(q) \mid Z_{S}\left(p^{\prime}\right)\right\rangle \\
& \times \frac{\left\langle Z_{S}\left(p^{\prime}\right)\left|J^{\dagger}\right| 0\right\rangle}{p^{\prime 2}-m_{Z_{S}}^{2}} \ldots
\end{aligned}
$$

By introducing the matrix elements

$$
\begin{aligned}
& \left\langle 0\left|J^{D_{s}}\right| D_{s}(p)\right\rangle=\frac{m_{D_{s}}^{2} f_{D_{s}}}{m_{c}+m_{s}}, \\
& \left\langle D_{s}(p) \pi(q) \mid Z_{S}\left(p^{\prime}\right)\right\rangle=g_{Z_{S} D_{s} \pi} p \cdot p^{\prime},
\end{aligned}
$$

we can rewrite $\Pi^{\text {Phys }}(p, q)$ in the form

$\Pi^{\mathrm{Phys}}(p, q)=\frac{g_{Z_{S} D_{s} \pi} m_{D_{s}}^{2} m_{Z_{S}} f_{D_{s}} f_{Z_{S}}}{\left(p^{2}-m_{D_{s}}^{2}\right)\left(p^{\prime 2}-m_{Z_{S}}^{2}\right)\left(m_{c}+m_{s}\right)} p \cdot p^{\prime}+\cdots$

Here $m_{D_{s}}$ and $f_{D_{s}}$ are the mass and decay constant of the meson $D_{s}$, respectively.
In terms of the quark-gluon degrees of freedom $\Pi(p, q)$ is given by the expression

$$
\begin{aligned}
\Pi^{\mathrm{QCD}}(p, q)= & i \int d^{4} x e^{i p x}\left\{\left[\gamma_{5} \widetilde{S}_{s}^{i a}(x) \gamma_{5} \widetilde{S}_{c}^{b i}(-x) \gamma_{5}\right]_{\alpha \beta}\right. \\
& \times\left\langle\pi(q)\left|\bar{d}_{\alpha}^{b}(0) u_{\beta}^{a}(0)\right| 0\right\rangle \\
& -\left[\gamma_{5} \widetilde{S}_{s}^{i a}(x) \gamma_{5} \widetilde{S}_{c}^{a i}(-x) \gamma_{5}\right] \\
& \left.\times\left\langle\pi(q)\left|\bar{d}_{\alpha}^{b}(0) u_{\beta}^{b}(0)\right| 0\right\rangle\right\}
\end{aligned}
$$

where $\alpha$ and $\beta$ are the spinor indices. To continue we employ the expansion

$\bar{u}_{\alpha}^{a} d_{\beta}^{d} \rightarrow \frac{1}{4} \Gamma_{\beta \alpha}^{j}\left(\bar{u}^{a} \Gamma^{j} d^{d}\right)$,

with $\Gamma^{j}$ being the full set of Dirac matrices

$\Gamma^{j}=\mathbf{1}, \gamma_{5}, \gamma_{\lambda}, i \gamma_{5} \gamma_{\lambda}, \sigma_{\lambda \rho} / \sqrt{2}$

The operators $\bar{u}^{a}(0) \Gamma^{j} d^{d}(0)$, as well as three-particle operators that appear due to insertion of $G_{\mu \nu}$ from propagators $\widetilde{S}_{s}(x)$ and $\widetilde{S}_{c}(-x)$ into $\bar{u}^{a}(0) \Gamma^{j} d^{d}(0)$ give rise to local matrix elements of the pion. In other words, instead of the distribution amplitudes the function $\Pi^{\mathrm{QCD}}(p, q)$ depends on the pion's local matrix elements. Then, the conservation of four-momentum in the tetraquark-meson-meson vertex can be obeyed by setting $q=0$. In the limit $q \rightarrow 0$ we get $p=p^{\prime}$ and have to carry out Borel transformations over one variable 
$p^{2}$. This condition has to be implemented in the physical side of the sum rule, as well $[43,46]$.

After substituting Eq. (29) into the expression of the correlation function and performing the summation over color indices in accordance with recipes presented in a detailed form in Ref. [43], we fix local matrix elements that enter to $\Pi^{\mathrm{QCD}}(p, q)$. It turns out that only the matrix element of the pion

$$
\left\langle 0\left|\bar{d}(0) i \gamma_{5} u(0)\right| \pi(q)\right\rangle=f_{\pi} \mu_{\pi},
$$

where $\mu_{\pi}=-2\langle\bar{q} q\rangle / f_{\pi}^{2}$ contributes to the correlation function. Other matrix elements including three-particle ones either do not contribute to a final expression of $\Pi^{\mathrm{QCD}}(p, q)$ or vanish in the soft limit $q \rightarrow 0$.

In the soft limit the Borel transformation of relevant invariant function $\Pi^{\mathrm{QCD}}\left(p^{2}\right)$ can be obtained after the following operations: we find the spectral density $\rho^{\text {pert. }}(s)$ as imaginary part of $\Pi^{\text {pert. }}\left(p^{2}\right)$, which is the perturbative component of the full correlation function. It is calculated using Eq. (28) and keeping in the quark propagators only their perturbative components. All other terms in $\Pi^{\mathrm{QCD}}\left(p^{2}\right)$ constitute the nonperturbative peace of the correlator, i.e. function $\Pi^{\text {n.-pert. }}\left(p^{2}\right)$. We calculate Borel transformation of $\Pi^{\mathrm{n}}$--pert. $\left(p^{2}\right)$ directly from Eq. (28) in accordance with prescriptions of Ref. [41], and by this way bypass intermediate steps, i.e. computation of $\rho^{\text {n.-pert. }}(s)$, which becomes unnecessary in this case. This approach considerably simplifies calculations and allows us to find explicitly $\Pi^{\text {n.-pert. }}\left(M^{2}\right) \equiv \mathcal{B} \Pi^{\text {n. }- \text { pert. }}\left(p^{2}\right)$. For the spectral density $\rho^{\text {pert. }}(s)$ we obtain

$\rho^{\text {pert. }}(s)=\frac{f_{\pi} \mu_{\pi}}{16 \pi^{2} s}\left(s-m_{c}^{2}\right)\left(s+2 m_{c} m_{s}-m_{c}^{2}\right)$.

The Borel transformed $\Pi^{\text {n.- }}$ pert. $\left(M^{2}\right)$ contains terms up to nine dimensions and reads

$$
\Pi^{\mathrm{n} .- \text { pert. }}\left(M^{2}\right)=\frac{f_{\pi} \mu_{\pi}}{12 M^{2}} e^{-m_{c}^{2} / M^{2}} \sum_{l=1}^{5} F_{l}\left(M^{2}\right),
$$

where

$$
\begin{aligned}
F_{1}\left(M^{2}\right)= & -\langle\bar{s} s\rangle\left(m_{c}^{2} m_{s}+2 m_{c} M^{2}-m_{s} M^{2}\right), \\
F_{2}\left(M^{2}\right)= & \frac{m_{c}}{12 M^{2}}\left\langle\frac{\alpha_{s} G^{2}}{\pi}\right\rangle \int_{0}^{1} \frac{d z e^{m_{c}^{2} / M^{2}-m_{c}^{2} /\left[M^{2} z(1-z)\right]}}{z(z-1)^{3}} \\
& \times\left[m_{c}^{3} z+2 m_{s} M^{2}(1-z)^{2} z+m_{c}^{2} m_{s}(1-z)\right], \\
F_{3}\left(M^{2}\right)= & \frac{m_{c}^{3}}{6 M^{4}}\left\langle\bar{s} g_{s} \sigma G s\right\rangle\left(m_{c} m_{s}+3 M^{2}\right), \\
F_{4}\left(M^{2}\right)= & -\frac{m_{c} \pi^{2}}{18 M^{6}}\left\langle\frac{\alpha_{s} G^{2}}{\pi}\right\rangle\langle\bar{s} s\rangle\left[m_{c}^{3} m_{s}\right. \\
& \left.+2 m_{c} M^{2}\left(m_{c}+m_{s}\right)+6 M^{4}\right],
\end{aligned}
$$

$$
\begin{aligned}
F_{5}\left(M^{2}\right)= & \frac{m_{c} \pi^{2}}{108 M^{10}}\left\langle\frac{\alpha_{s} G^{2}}{\pi}\right\rangle\left\langle\bar{s} g_{s} \sigma G s\right\rangle\left(m_{c} m_{s}+3 M^{2}\right) \\
& \times\left(m_{c}^{4}+6 m_{c}^{2} M^{2}+6 M^{4}\right) .
\end{aligned}
$$

Then in the soft limit the Borel transformation of $\Pi^{\mathrm{QCD}}\left(p^{2}\right)$ takes the form

$$
\begin{aligned}
\mathcal{B} \Pi^{\mathrm{QCD}}\left(p^{2}\right)= & \int_{\left(m_{c}+m_{s}\right)^{2}}^{\infty} \rho^{\text {pert. }}(s) e^{-s / M^{2}} d s \\
& +\Pi^{\text {n. }- \text { pert. }}\left(M^{2}\right) .
\end{aligned}
$$

In the same limit the Borel transformation of $\Pi^{\mathrm{Phys}}\left(p^{2}\right)$ is given by the expression

$\mathcal{B} \Pi^{\text {Phys }}\left(p^{2}\right)=\frac{g_{Z_{S} D_{s} \pi} m_{D_{s}}^{2} m_{Z_{S}} f_{D_{s}} f_{Z_{S}}}{m_{c}+m_{s}} m^{2} \frac{e^{-m^{2} / M^{2}}}{M^{2}}+\cdots$,

where $m^{2}=\left(m_{D_{s}}^{2}+m_{Z_{S}}^{2}\right) / 2$.

After equating $\mathcal{B} \Pi^{\text {Phys }}\left(p^{2}\right)$ and $\mathcal{B} \Pi^{\mathrm{QCD}}\left(p^{2}\right)$ one has to subtract contributions of higher resonances and continuum states. In the case of standard sum rules (i.e. $q \neq 0$ ) this can be carried out quite easily, because Borel transformation suppress all undesired terms in the physical side of the equality. But in the soft limit $\mathcal{B} \Pi^{\text {Phys }}\left(p^{2}\right)$ contains terms which are not suppressed even after Borel transformation [41], therefore additional manipulations are required to remove them from the phenomenological side of the sum rule. Acting by the operator

$\mathcal{P}\left(M^{2}, m^{2}\right)=\left(1-M^{2} \frac{d}{d M^{2}}\right) M^{2} e^{m^{2} / M^{2}}$

one can achieve this goal [42]. Then subtraction can be performed in a standard manner and leads to the sum rule

$$
\begin{aligned}
g_{Z_{S} D_{s} \pi}= & \frac{m_{c}+m_{s}}{m_{D_{s}}^{2} m_{Z_{S}} m^{2} f_{D_{s}} f_{Z_{S}}} \mathcal{P}\left(M^{2}, m^{2}\right) \\
& \times\left[\int_{\left(m_{c}+m_{s}\right)^{2}}^{s_{0}} \rho^{\text {pert. }}(s) e^{-s / M^{2}} d s\right. \\
& \left.+\Pi^{\text {n. - pert. }}\left(M^{2}\right)\right] .
\end{aligned}
$$

It is worth noting that we do not perform continuum subtractionin in nonperturbative terms $\sim\left(M^{2}\right)^{0}$ and $\sim$ $\left(M^{2}\right)^{-n}, n=1,2 \ldots$ [41].

With the coupling $g_{Z_{S} D_{s} \pi}$ at hands it is straightforward to evaluate the width of the decay $Z_{S} \rightarrow D_{s} \pi$

$$
\begin{aligned}
\Gamma\left(Z_{S} \rightarrow D_{s} \pi\right)= & \frac{g_{Z_{S} D_{s} \pi}^{2}}{96 \pi m_{Z_{S}}^{2}}\left(m_{Z_{S}}^{2}+m_{D_{s}}^{2}-m_{\pi}^{2}\right)^{2} \\
& \times f\left(m_{Z_{S}}, m_{D_{s}}, m_{\pi}\right),
\end{aligned}
$$

where the function $f(x, y, z)$ is

$$
f(x, y, z)=\frac{1}{2 x} \sqrt{x^{4}+y^{4}+z^{4}-2 x^{2} y^{2}-2 x^{2} z^{2}-2 y^{2} z^{2}} .
$$


Another decay channel of the doubly charged charmstrange tetraquark is $Z_{S} \rightarrow D K$. Correlation function that should be considered in this case is given by the expression

$\Pi_{K}(p, q)=i \int d^{4} x e^{i p x}\left\langle K(q)\left|\mathcal{T}\left\{J^{D}(x) J^{\dagger}(0)\right\}\right| 0\right\rangle$,

where the interpolating current for $D$ meson is

$J^{D}(x)=\bar{c}_{i}(x) \gamma_{5} d_{i}(x)$

The analysis of the channel $Z_{S} \rightarrow D K$ does not differ considerably from consideration of $Z_{S} \rightarrow D_{S} \pi$ decay. Because particles in final states $D_{s} \pi$ and $D K$ are pseudoscalar mesons, differences between two decay channels are encoded in the matrix element

$\left\langle 0\left|J^{D}\right| D(p)\right\rangle=\frac{m_{D}^{2} f_{D}}{m_{c}+m_{d}}$,

and local matrix element of $K$ meson

$\left\langle 0\left|\bar{u}(0) i \gamma_{5} s(0)\right| K(q)\right\rangle=\frac{f_{K} m_{K}^{2}}{m_{s}+m_{u}}$,

that contributes to $\Pi_{K}^{\mathrm{QCD}}(p, q)$, where $m_{K}$ and $f_{K}$ are the mass and decay constant of $K$ meson. The strong coupling $g_{Z_{S} D K}$ with evident replacements is defined by Eq. (27).

In the $P$-wave decay $Z_{S} \rightarrow D_{S 1}(2460) \pi$ the interpolating current, matrix element and strong coupling of the axial-vector meson $D_{s 1}(2460)$ are introduced by means of the formulas

$J_{\mu}^{D_{s 1}}(x)=\bar{c}_{i}(x) \gamma_{\mu} \gamma_{5} s_{i}(x)$,

$\left\langle 0\left|J_{\mu}^{D_{s 1}}\right| D_{s 1}(p)\right\rangle=f_{D_{s 1}} m_{D_{s 1}} \varepsilon_{\mu}$,

$\left\langle D_{s 1}(p) \pi(q) \mid Z_{S}\left(p^{\prime}\right)\right\rangle=g_{Z_{S} D_{s 1} \pi} \varepsilon^{*} \cdot p^{\prime}$,

with $m_{D_{s 1}}, f_{D_{s 1}}$ and $\varepsilon_{\mu}$ being its mass, decay constant and polarization vector, respectively.

The remaining operations and intermediate steps in both cases are standard ones, therefore we refrain from presenting them here in a detailed form, and write down only formula for the decay width $\Gamma\left(Z_{S} \rightarrow D_{s 1}(2460) \pi\right)$ :

$\Gamma\left(Z_{S} \rightarrow D_{s 1}(2460) \pi\right)=\frac{g_{Z_{S} D_{s 1} \pi}^{2}}{24 \pi m_{D_{s 1}}^{2}} f^{3}\left(m_{Z_{S}}, m_{D_{s 1}}, m_{\pi}\right)$.

Numerical calculations are carried out using the sum rules derived for strong couplings and expressions for widths of different decay modes of $Z_{S}$. The masses and decay constants of $D_{s}, D$ and $D_{s 1}(2460)$, as well as $\pi$ and $K$ mesons which we employ in numerical computations are collected in Table 2. The masses of particles are taken from Ref. [48], for decay constants of $D$ and $D_{s}$ mesons we use information from Ref. [49], decay constant of $D_{s 1}(2460)$ is borrowed
Table 2 Parameters of the mesons used in numerical calculations

\begin{tabular}{ll}
\hline Parameters & Values (Mev) \\
\hline$m_{D}$ & $(1869.5 \pm 0.4)$ \\
$f_{D}$ & $(211.9 \pm 1.1)$ \\
$m_{D_{s}}$ & $(1969.0 \pm 1.4)$ \\
$f_{D_{s}}$ & $(249.0 \pm 1.2)$ \\
$m_{D_{s 1}}$ & $(2459.6 \pm 0.9)$ \\
$f_{D_{s 1}}$ & $(481 \pm 164)$ \\
$m_{D_{s}^{*}}$ & $(2112.1 \pm 0.4)$ \\
$f_{D_{s}^{*}}$ & $(308 \pm 21)$ \\
$m_{D^{*}}$ & $(2010.26 \pm 0.25)$ \\
$f_{D^{*}}$ & $(252.2 \pm 22.66)$ \\
$m_{D_{s 0}^{*}}$ & $(2318.0 \pm 1.0)$ \\
$f_{D_{s 0}^{*}}$ & 201 \\
$m_{K}$ & $(493.677 \pm 0.016)$ \\
$f_{K}$ & 156 \\
$m_{\pi}$ & $(139.57061 \pm 0.00024)$ \\
$f_{\pi}$ & 131 \\
\hline
\end{tabular}

from [50]. Table 2 contains also parameters of the $D^{*}, D_{s}^{*}$ and $D_{s 0}^{*}(2317)$ mesons which will be used in the next section.

The Borel parameter $M^{2}$ and continuum threshold $s_{0}$ in coupling calculations are chosen as in Eq. (22). For the strong couplings of the explored vertices and width of the decay modes we obtain: for the channel $Z_{S} \rightarrow D_{s} \pi$

$g_{Z_{S} D_{s} \pi}=(0.51 \pm 0.14) \mathrm{GeV}^{-1}$

$\Gamma\left(Z_{S} \rightarrow D_{S} \pi\right)=(8.27 \pm 2.32) \mathrm{MeV}$,

for the mode $Z_{S} \rightarrow D K$

$g_{Z_{S} D K}=(1.54 \pm 0.43) \mathrm{GeV}^{-1}$,

$\Gamma\left(Z_{S} \rightarrow D K\right)=(57.41 \pm 14.93) \mathrm{MeV}$,

and for $Z_{S} \rightarrow D_{s 1}(2460) \pi$

$g_{Z_{S} D s 1 \pi}=26.16 \pm 7.36$,

$\Gamma\left(Z_{S} \rightarrow D_{s 1}(2460) \pi\right)=(1.21 \pm 0.38) \mathrm{MeV}$.

The full width of the scalar tetraquark $Z_{S}$ on the basis of considered decay modes is equal to

$\Gamma_{Z_{S}}=(66.89 \pm 15.11) \mathrm{MeV}$,

which is typical for a diquark-antidiquark state: The tetraquark $Z_{S}$ belongs neither to a class of broad resonances $\Gamma \sim$ $200 \mathrm{MeV}$ nor to a class of very narrow states $\Gamma \sim 1 \mathrm{MeV}$.

The charmed particle composed of four different quarks as a partner of the $X(5568)$ resonance was previously investigated in our work [38]. We analyzed this state using the interpolating currents of both $C \gamma_{5} \otimes \gamma_{5} C$ and $C \gamma_{\mu} \otimes \gamma^{\mu} C$ types. The diquark-antidiquark composition of $X_{c}=[s u][\bar{c} \bar{d}]$ 
means that it is a neutral particle. Nevertheless, it is instructive to compare parameters of $X_{c}$ with results for $Z_{S}$ obtained in the present work. In the case of the interpolating current $C \gamma_{5} \otimes \gamma_{5} C$ we found $m_{X_{c}}=(2634 \pm 62) \mathrm{MeV}$ which is very close to our present result. The processes $X_{c} \rightarrow \bar{D}^{0} \bar{K}^{0}$ and $X_{c} \rightarrow D_{s}^{-} \pi^{+}$were also subject of studies in Ref. [38]. Width of these decay channels $\Gamma\left(X_{c} \rightarrow \bar{D}^{0} \bar{K}^{0}\right)=$ (53.7 \pm 11.6$) \mathrm{MeV}$ and $\Gamma\left(X_{c} \rightarrow D_{s}^{-} \pi^{+}\right)=(8.2 \pm 2.1) \mathrm{MeV}$ are comparable with ones presented in Eqs. (44) and (45).

\section{$4 Z_{P S} \rightarrow D_{s}^{*} \pi, D^{*} K, D_{s 0}^{*}(2317) \pi$ and \\ $Z_{A V} \rightarrow D_{s}^{*} \pi, D^{*} K, D_{s 1}(2460) \pi$ decays of the pseudoscalar and axial-vector tetraquarks}

The pseudoscalar $Z_{P S}$ and axial-vector $Z_{A V}$ tetraquarks may decay through different channels. Among kinematically allowed decay channels of $Z_{P S}$ state are $S$-wave mode $Z_{P S} \rightarrow D_{S 0}(2317) \pi$, and $P$-wave modes $Z_{P S} \rightarrow$ $D_{S}^{*} \pi$ and $D^{*} K$. The decays of the tetraquark $Z_{A V}$ include $S$-wave channels $Z_{A V} \rightarrow D_{S}^{*} \pi, D^{*} K$ and $P$-wave mode $Z_{A V} \rightarrow D_{s 1}(2460) \pi$.

It is seen that both $Z_{P S}$ and $Z_{A V}$ states decay to $D_{s}^{*} \pi$ and $D^{*} K$, therefore these channels should be analyzed in a connected form. We start our investigation from analysis of the decays $Z_{A V} \rightarrow D_{s}^{*} \pi$ and $Z_{P S} \rightarrow D_{S}^{*} \pi$, and construct the following correlation function

$\Pi_{\mu \nu}(p, q)=i \int d^{4} x e^{i p x}\left\langle\pi(q)\left|\mathcal{T}\left\{J_{\mu}^{D_{s}^{*}}(x) J_{\nu}^{\dagger}(0)\right\}\right| 0\right\rangle$,

where $J_{\mu}^{D_{s}^{*}}(x)$ is the interpolating current of the $D_{s}^{*}$ meson

$J_{\mu}^{D_{s}^{*}}(x)=\bar{c}_{l}(x) \gamma_{\mu} s_{l}(x)$.

The function $\Pi_{\mu \nu}(p, q)$ will be computed employing QCD sum rule on the light-cone and using a technique of the softmeson approximation. Because the current $J_{v}(x)$ couples to both the pseudoscalar and axial-vector tetraquarks the correlator $\Pi_{\mu \nu}^{\text {Phys }}(p, q)$ expressed in terms of the physical parameters of the involved particles and vertices contains two components: Indeed, for $\Pi_{\mu \nu}^{\text {Phys }}(p, q)$ we find:

$$
\begin{aligned}
\Pi_{\mu \nu}^{\text {Phys }}(p, q)= & \frac{\left\langle 0\left|J_{\mu}^{D_{s}^{*}}\right| D_{s}^{*}(p)\right\rangle}{p^{2}-m_{D_{s}^{*}}^{2}}\left\langle D_{s}^{*}(p) \pi(q) \mid Z_{P S}\left(p^{\prime}\right)\right\rangle \\
& \times \frac{\left\langle Z_{P S}\left(p^{\prime}\right)\left|J_{v}^{\dagger}\right| 0\right\rangle}{p^{\prime 2}-m_{Z_{P S}}^{2}} \\
& +\frac{\left\langle 0\left|J_{\mu}^{D_{s}^{*}}\right| D_{s}^{*}(p)\right\rangle}{p^{2}-m_{D_{s}^{*}}^{2}}\left\langle D_{s}^{*}(p) \pi(q) \mid Z_{A V}\left(p^{\prime}\right)\right\rangle \\
& \times \frac{\left\langle Z_{A V}\left(p^{\prime}\right)\left|J_{v}^{\dagger}\right| 0\right\rangle}{p^{\prime 2}-m_{Z_{A V}}^{2}} \ldots
\end{aligned}
$$

The terms in Eq. (49) are contributions of vertices $Z_{P S} D_{s}^{*} \pi$ and $Z_{A V} D_{s}^{*} \pi$, where all particles are on their ground states. The dots stand for effects due to the higher resonances and continuum.

We introduce the $D_{s}^{*}$ meson matrix element

$\left\langle 0\left|J_{\mu}^{D_{s}^{*}}\right| D_{s}^{*}(p)\right\rangle=f_{D_{s}^{*} m_{D_{s}^{*}} \varepsilon_{\mu}}$,

where $m_{D_{s}^{*}}, f_{D_{s}^{*}}$ and $\varepsilon_{\mu}$ are its mass, decay constant and polarization vector, respectively. We define also the matrix elements corresponding to the vertices in the following manner

$$
\begin{aligned}
\left\langle D_{s}^{*}(p) \pi(q) \mid Z_{A V}\left(p^{\prime}\right)\right\rangle= & g_{Z_{A V} D_{s}^{*} \pi}\left[\left(p \cdot p^{\prime}\right)\left(\varepsilon^{*} \cdot \varepsilon^{\prime}\right)\right. \\
& \left.-\left(q \cdot \varepsilon^{\prime}\right)\left(p^{\prime} \cdot \varepsilon^{*}\right)\right],
\end{aligned}
$$

and

$\left\langle D_{s}^{*}(p) \pi(q) \mid Z_{P S}\left(p^{\prime}\right)\right\rangle=g_{Z_{P S} D_{s}^{*} \pi} p^{\prime} \cdot \varepsilon$.

After some manipulations the ground state terms in $\Pi_{\mu \nu}^{\text {Phys }}(p, q)$ can be easily rewritten as:

$$
\begin{aligned}
\Pi_{\mu \nu}^{\text {Phys }}(p, q)= & g_{Z_{A V} D_{s}^{*} \pi} \frac{m_{D_{s}^{*}} f_{D_{s}^{*}} m_{Z_{A V}} f_{Z_{A V}}}{\left(p^{2}-m_{D_{s}^{*}}^{2}\right)\left(p^{\prime 2}-m_{Z_{A V}}^{2}\right)} \\
& \times\left(\frac{m_{Z_{A V}}^{2}+m_{D_{s}^{*}}^{2}}{2} g_{\mu \nu}-p_{\mu} p_{v}^{\prime}\right) \\
& +\frac{g_{Z_{P S} D_{s}^{*} \pi} f_{D_{s}^{*}} m_{Z_{P S}} f_{Z_{P S}}}{\left(p^{2}-m_{D_{s}^{*}}^{2}\right)\left(p^{2}-m_{Z_{P S}}^{2}\right) m_{D_{s}^{*}}} \\
& \times \frac{m_{Z_{P S}}^{2}-m_{D_{s}^{*}}^{2}}{2} p_{\mu} p_{v}^{\prime}+\cdots
\end{aligned}
$$

One sees that $\Pi_{\mu \nu}^{\text {Phys }}(p, q)$ contains two structures $\sim g_{\mu \nu}$ and $\sim p_{\mu} p_{v}^{\prime}$. The same structures appear in the second part of the sum rule which is the correlation function Eq. (47) calculated in terms of quark propagators. For $\Pi_{\mu \nu}^{\mathrm{QCD}}(p, q)$ we get

$$
\begin{aligned}
& \Pi_{\mu \nu}^{\mathrm{QCD}}(p, q)=i \int d^{4} x e^{i p x}\left\{\left[\gamma_{\nu} \widetilde{S}_{s}^{i a}(x) \gamma_{\mu}\right.\right. \\
& \left.\quad \times \widetilde{S}_{c}^{b i}(-x) \gamma_{5}\right]_{\alpha \beta}\left\langle\pi(q)\left|\bar{d}_{\alpha}^{b}(0) u_{\beta}^{a}(0)\right| 0\right\rangle \\
& \left.-\left[\gamma_{\nu} \widetilde{S}_{s}^{i a}(x) \gamma_{\mu} \widetilde{S}_{c}^{a i}(-x) \gamma_{5}\right]\left\langle\pi(q)\left|\bar{d}_{\alpha}^{b}(0) u_{\beta}^{b}(0)\right| 0\right\rangle\right\} .
\end{aligned}
$$

We use invariant amplitudes corresponding to structures $\sim g_{\mu \nu}$ from $\Pi_{\mu \nu}^{\text {Phys }}(p, q)$ and $\Pi_{\mu \nu}^{\mathrm{QCD}}(p, q)$ to derive sum rule for the coupling $g_{Z_{A V} D_{s}^{*} \pi}$. To this end, we equate these invariant amplitudes and carry out calculations in accordance with scheme described in rather detailed form in the previous section. Obtained by this way sum rule is employed to evaluate the strong coupling $g_{Z_{A V} D_{s}^{*} \pi}$. It is utilized as an input parameter at the second stage of analysis, when we employ invariant amplitudes corresponding to structures $\sim p_{\mu} p_{\nu}^{\prime}$ to derive sum rule for $g_{Z_{P S} D_{s}^{*} \pi}$. 
The decays $Z_{A V} \rightarrow D^{*} K$ and $Z_{P S} \rightarrow D^{*} K$ can be investigated in the same way, but one has to start from the correlator

$\Pi_{\mu \nu}(p, q)=i \int d^{4} x e^{i p x}\left\langle K(q)\left|\mathcal{T}\left\{J_{\mu}^{D^{*}}(x) J_{\nu}^{\dagger}(0)\right\}\right| 0\right\rangle$,

with $J_{\mu}^{D^{*}}(x)$

$J_{\mu}^{D^{*}}(x)=\bar{c}_{l}(x) \gamma_{\mu} d_{l}(x)$

The remaining analysis does not differ from calculations of the decays $Z_{A V} \rightarrow D_{S}^{*} \pi$ and $Z_{P S} \rightarrow D_{S}^{*} \pi$, and therefore we do not provide further details.

There are also two processes $Z_{P S} \rightarrow D_{s 0}^{*}(2317) \pi$ and $Z_{A V} \rightarrow D_{s 1}(2460) \pi$ which are not connected with each other, and can be studied separately. Let us consider, for example, decay $Z_{P S} \rightarrow D_{s 0}^{*}(2317) \pi$ that can be explored by means of the correlator

$\Pi_{v}(p, q)=i \int d^{4} x e^{i p x}\left\langle\pi(q)\left|\mathcal{T}\left\{J^{D_{s 0}^{*}}(x) J_{v}^{\dagger}(0)\right\}\right| 0\right\rangle$,

where the interpolating current $J_{\mu}^{D_{s 0}^{*}}(x)$ is chosen in the form

$J^{D_{s 0}^{*}}(x)=\bar{c}^{i}(x) s^{i}(x)$.

The correlation function $\Pi_{v}(p, q)$ has the following phenomenological representation

$$
\begin{aligned}
\Pi_{\nu}^{\text {Phys }}(p, q)= & \frac{\left\langle 0\left|J^{D_{s 0}^{*}}\right| D_{s 0}^{*}(p)\right\rangle}{p^{2}-m_{D_{s 0}^{2}}^{2}}\left\langle D_{s 0}^{*}(p) \pi(q) \mid Z_{P S}\left(p^{\prime}\right)\right\rangle \\
& \times \frac{\left\langle Z_{P S}\left(p^{\prime}\right)\left|J_{v}^{\dagger}\right| 0\right\rangle}{p^{\prime 2}-m_{Z_{P S}}^{2}}+\cdots
\end{aligned}
$$

Using of the matrix element

$\left\langle 0\left|J^{D_{s 0}^{*}}\right| D_{s 0}^{*}(p)\right\rangle=f_{D_{s 0}^{*}} m_{D_{s 0}^{*}}$,

and also the vertex

$\left\langle D_{s 0}^{*}(p) \pi(q) \mid Z_{P S}\left(p^{\prime}\right)\right\rangle=g_{Z_{P S} D_{s 0}^{*} \pi} p \cdot p^{\prime}$,

it can be rewritten as

$\Pi_{v}^{\text {Phys }}(p, q)=g_{Z_{P S} D_{s 0}^{*} \pi} \frac{f_{Z_{P S}} m_{Z_{P S}} f_{D_{s 0}^{*}} m_{D_{s 0}^{*}}}{\left(p^{2}-m^{2}\right)^{2}} m^{2} p_{v}^{\prime}+\cdots$,

where $m^{2}=\left(m_{Z_{P S}}^{2}+m_{D_{s 0}^{*}}^{2}\right) / 2$. In order to match the obtained expression with the same structure from $\Pi_{v}^{\mathrm{QCD}}(p, q)$ we keep in Eq. (61) dependence on $p_{v}^{\prime}$, whereas in the invariant amplitude, i.e. in the function $\sim p_{v}^{\prime}$ implement the soft limit.
Table 3 The strong couplings and decay widths of the $Z_{A V}$ and $Z_{P S}$ tetraquarks

\begin{tabular}{lll}
\hline Decay & Strong couplings & Decay width (Mev) \\
\hline$Z_{A V} \rightarrow D_{s}^{*} \pi$ & $(0.26 \pm 0.07) \mathrm{GeV}^{-1}$ & $(7.94 \pm 2.21)$ \\
$Z_{A V} \rightarrow D^{*} K$ & $(0.63 \pm 0.17) \mathrm{GeV}^{-1}$ & $(37.38 \pm 10.84)$ \\
$Z_{A V} \rightarrow D_{S 1} \pi$ & $(1.55 \pm 0.43) \mathrm{GeV}^{-1}$ & $(2.02 \pm 0.59)$ \\
$Z_{P S} \rightarrow D_{s}^{*} \pi$ & $3.18 \pm 0.94$ & $(4.37 \pm 1.27)$ \\
$Z_{P S} \rightarrow D^{*} K$ & $8.24 \pm 2.39$ & $(19.09 \pm 5.73)$ \\
$Z_{P S} \rightarrow D_{s 0}^{*} \pi$ & $(0.76 \pm 0.18) \mathrm{GeV}^{-1}$ & $(14.64 \pm 3.94)$ \\
\hline
\end{tabular}

The same correlation function $\Pi_{v}(p, q)$ in terms of quark propagators and pion's matrix elements is given by formula

$$
\begin{aligned}
\Pi_{v}^{\mathrm{QCD}}(p, q)= & i \int d^{4} x e^{i p x}\left\{\left[\gamma_{\nu} \widetilde{S}_{s}^{i a}(x) \widetilde{S}_{c}^{b i}(-x) \gamma_{5}\right]_{\alpha \beta}\right. \\
& \times\left\langle\pi(q)\left|\bar{d}_{\alpha}^{b}(0) u_{\beta}^{a}(0)\right| 0\right\rangle \\
& -\left[\gamma_{\nu} \widetilde{S}_{s}^{i a}(x) \tilde{S}_{c}^{a i}(-x) \gamma_{5}\right] \\
& \left.\times\left\langle\pi(q)\left|\bar{d}_{\alpha}^{b}(0) u_{\beta}^{b}(0)\right| 0\right\rangle\right\}
\end{aligned}
$$

After calculations one finds that in $\Pi_{v}^{\mathrm{QCD}}(p, q)$ survives only the structure $\sim p_{v}^{\prime}$. By equating invariant amplitudes from both sides and performing all manipulations it is possible to derive the sum rule for the coupling $g_{Z_{P S} D_{s 0}^{*} \pi}$. The similar analysis has been carried out for the decay $Z_{A V} \rightarrow$ $D_{s 1}(2460) \pi$, as well.

In numerical calculations of the $Z_{P S}$ and $Z_{A V}$ states' strong couplings the Borel parameter and continuum threshold are chosen within the same ranges as in computations of their masses (see, Table 1). As input parameters we employ also mass and decay constant of the mesons $D_{s}^{*}, D^{*}$ and $D_{s 0}^{*}(2317)$ from Table 2. It is worth noting that the decay constants $f_{D_{s}^{*}}, f_{D^{*}}$ and $f_{D_{s 0}^{*}}$ have been taken from Refs. [51-53], respectively.

Results for strong couplings and width of decay modes of $Z_{P S}$ and $Z_{A V}$ tetraquarks are presented in Table 3. Using these predictions one can evaluate full widths of the pseudoscalar and axial-vector tetraquarks $Z_{P S}$ and $Z_{A V}$ :

$\Gamma_{Z_{P S}}=(38.1 \pm 7.1) \mathrm{MeV}$,

and

$\Gamma_{Z_{A V}}=(47.3 \pm 11.1) \mathrm{MeV}$.

As is seen, the tetraquarks $Z_{P S}$ and $Z_{A V}$ are narrower than the scalar state $Z_{S}$. Nevertheless, we cannot classify them as narrow resonances. 


\section{Conclusions}

In the present work we have investigated the charm-strange tetraquarks $Z_{\bar{c} s}=[s d][\overline{u c}]$ by calculating their spectroscopic parameters and decay channels. It is easy to see that these states bear two units of electric charge $-|e|$ and belong to a class of doubly charged tetraquarks. Their counterparts with the structure $Z_{c \bar{s}}=[u c][\bar{s} \bar{d}]$ have evidently a charge $+2|e|$. We have considered scalar, pseudoscalar and axial-vector doubly charged states. Their masses have been obtained using QCD two-point sum rule method. Our results have allowed us to fix possible decay channels of these states and found their widths. Investigations confirm that the doubly charged diquark-antidiquarks are neither broad states nor very narrow resonances.

Observation of doubly charged tetraquarks may open new stage in exploration of multiquark systems. In fact, resonances that are interpreted as hidden charm (bottom) tetraquarks may be also considered as excited states of charmonia (bottomonia) or their superpositions. The charged resonances can not be explained by this way, and are serious candidates to genuine tetraquarks. They may have diquark-antidiquark structure or be bound states of conventional mesons. In the last case, charged and neutral conventional mesons create shallow molecular states with large decay width. Therefore, it is reasonable to assume that doubly charged tetraquarks presumably exist only as diquarkantidiquarks, because binding of two mesons with the same electric charge to form a molecular state due to repulsive forces between them seems problematic.

The doubly charged tetraquarks deserve further detailed investigations. These studies should embrace also $Z_{b \bar{c}}$-type states that constitute a subclass of open charm-bottom states. Experimental exploration and discovery of $Z_{c \bar{s}}$ and/or $Z_{b \bar{c}}$ tetraquarks may have far-reaching consequences for hadron spectroscopy.

Acknowledgements The work of S. S. A. was supported by Grant No. EIF-Mob-8-2017-4(30)-17/01/1 of the Science Development Foundation under the President of the Azerbaijan Republic. K. A. thanks TÜBITAK for the partial financial support provided under Grant No. $115 \mathrm{~F} 183$.

Open Access This article is distributed under the terms of the Creative Commons Attribution 4.0 International License (http://creativecomm ons.org/licenses/by/4.0/), which permits unrestricted use, distribution, and reproduction in any medium, provided you give appropriate credit to the original author(s) and the source, provide a link to the Creative Commons license, and indicate if changes were made.

Funded by SCOAP ${ }^{3}$.

\section{References}

1. S.K. Choi et al. [Belle Collaboration], Phys. Rev. Lett. 91, 262001 (2003)
2. V.M. Abazov et al. [D0 Collaboration], Phys. Rev. Lett. 93, 162002 (2004)

3. D. Acosta et al. [CDF Collaboration], Phys. Rev. Lett. 93, 072001 (2004)

4. B. Aubert et al. [BaBar Collaboration], Phys. Rev. D 71, 071103 (2005)

5. H.X. Chen, W. Chen, X. Liu, S.L. Zhu, Phys. Rep. 639, 1 (2016)

6. H.X. Chen, W. Chen, X. Liu, Y.R. Liu, S.L. Zhu, Rep. Prog. Phys. 80, 076201 (2017)

7. A. Esposito, A. Pilloni, A.D. Polosa, Phys. Rep. 668, 1 (2017)

8. A. Esposito, A.L. Guerrieri, F. Piccinini, A. Pilloni, A.D. Polosa, Int. J. Mod. Phys. A 30, 1530002 (2015)

9. C.A. Meyer, E.S. Swanson, Prog. Part. Nucl. Phys. 82, 21 (2015)

10. S.K. Choi et al. [Belle Collaboration], Phys. Rev. Lett. 100, 142001 (2008)

11. R. Mizuk et al. [Belle Collaboration], Phys. Rev. D 80, 031104 (2009)

12. K. Chilikin et al. [Belle Collaboration], Phys. Rev. D 88, 074026 (2013)

13. V.M. Abazov et al. [D0 Collaboration], Phys. Rev. Lett. 117, 022003 (2016)

14. The D0 Collaboration, D0 Note 6488-CONF (2016)

15. R. Aaij et al. [LHCb Collaboration], Phys. Rev. Lett. 117, 152003 (2016)

16. The CMS Collaboration, CMS PAS BPH-16-002 (2016)

17. S.S. Agaev, K. Azizi, H. Sundu, Phys. Rev. D 93, 074024 (2016)

18. W. Chen, H.X. Chen, X. Liu, T.G. Steele, S.L. Zhu, Phys. Rev. Lett. 117, 022002 (2016)

19. Z.G. Wang, Commun. Theor. Phys. 66, 335 (2016)

20. W. Wang, R. Zhu, Chin. Phys. C 40, 093101 (2016)

21. C.M. Zanetti, M. Nielsen, K.P. Khemchandani, Phys. Rev. D 93, 096011 (2016)

22. S.S. Agaev, K. Azizi, H. Sundu, Phys. Rev. D 93, 114007 (2016)

23. J.M. Dias, K.P. Khemchandani, A. Martinez Torres, M. Nielsen, C.M. Zanetti, Phys. Lett. B 758, 235 (2016)

24. Z.G. Wang, Eur. Phys. J. C 76, 279 (2016)

25. C.J. Xiao, D.Y. Chen, Eur. Phys. J. A 53, 127 (2017)

26. S.S. Agaev, K. Azizi, H. Sundu, Eur. Phys. J. Plus 131, 351 (2016)

27. T.J. Burns, E.S. Swanson, Phys. Lett. B 760, 627 (2016)

28. F.K. Guo, U.G. Meißner, B.S. Zou, Commun. Theor. Phys. 65, 593 (2016)

29. Q.F. Lu, Y.B. Dong, Phys. Rev. D 94, 094041 (2016)

30. M. Albaladejo, J. Nieves, E. Oset, Z.F. Sun, X. Liu, Phys. Lett. B 757, 515 (2016)

31. C.B. Lang, D. Mohler, S. Prelovsek, Phys. Rev. D 94, 074509 (2016)

32. A. Esposito, A. Pilloni, A.D. Polosa, Phys. Lett. B 758, 292 (2016)

33. X.W. Kang, J.A. Oller, Phys. Rev. D 94, 054010 (2016)

34. A. Esposito, M. Papinutto, A. Pilloni, A.D. Polosa, N. Tantalo, Phys. Rev. D 88, 054029 (2013)

35. A.L. Guerrieri, M. Papinutto, A. Pilloni, A.D. Polosa, N. Tantalo, PoS LATTICE 2014, 106 (2015)

36. W. Chen, H.X. Chen, X. Liu, T.G. Steele, S.L. Zhu, Phys. Rev. D 95(11), 114005 (2017)

37. D. Ebert, R.N. Faustov, V.O. Galkin, Phys. Lett. B 696, 241 (2011)

38. S.S. Agaev, K. Azizi, H. Sundu, Phys. Rev. D 93, 094006 (2016)

39. Y.R. Liu, X. Liu, S.L. Zhu, Phys. Rev. D 93, 074023 (2016)

40. I.I. Balitsky, V.M. Braun, A.V. Kolesnichenko, Nucl. Phys. B 312, 509 (1989)

41. V.M. Belyaev, V.M. Braun, A. Khodjamirian, R. Ruckl, Phys. Rev. D 51, 6177 (1995)

42. B.L. Ioffe, A.V. Smilga, Nucl. Phys. B 232, 109 (1984)

43. S.S. Agaev, K. Azizi, H. Sundu, Phys. Rev. D 93, 074002 (2016)

44. S.S. Agaev, K. Azizi, H. Sundu, Phys. Rev. D 95, 034008 (2017)

45. S.S. Agaev, K. Azizi, H. Sundu, Eur. Phys. J. C 77, 321 (2017)

46. S.S. Agaev, K. Azizi, H. Sundu, Phys. Rev. D 95, 114003 (2017) 
47. S.S. Agaev, K. Azizi, H. Sundu, Phys. Rev. D 96, 034026 (2017)

48. C. Patrignani et al. [Particle Data Group], Chin. Phys. C 40, 100001 (2016)

49. J.L. Rosner, S. Stone, R. S. Van de Water (2015). arXiv:1509.02220 [hep-ph]

50. J.Y. Sungu, H. Sundu, K. Azizi, N. Yinelek, S. Sahin, in The many faces of QCD, 1-5 Nov 2010. Ghent, Belgium, CNUM: C10-1101.1, PoS FACESQCD, 045 (2010)
51. S.S. Agaev, K. Azizi, H. Sundu, Phys. Rev. D 92, 116010 (2015)

52. W. Lucha, D. Melikhov, S. Simula, Phys. Lett. B 735, 12 (2014)

53. S. Narison, Phys. Lett. B 605, 319 (2005) 